\title{
Mass imbalances in EPANET water-quality simulations
}

\author{
Michael J. Davis ${ }^{1}$, Robert Janke ${ }^{2}$, and Thomas N. Taxon ${ }^{3}$ \\ ${ }^{1}$ Argonne Associate of Seville, Environmental Science Division, Argonne National Laboratory, \\ Argonne, Illinois, USA \\ ${ }^{2}$ National Homeland Security Research Center, US Environmental Protection Agency, Cincinnati, Ohio, USA \\ ${ }^{3}$ Global Security Sciences Division, Argonne National Laboratory, Argonne, Illinois, USA
}

Correspondence: Michael J. Davis (mike_davis@anl.gov) and Robert Janke (janke.robert@epa.gov)

Received: 2 September 2017 - Discussion started: 26 September 2017

Revised: 22 February 2018 - Accepted: 4 March 2018 - Published: 6 April 2018

\begin{abstract}
EPANET is widely employed to simulate water quality in water distribution systems. However, in general, the time-driven simulation approach used to determine concentrations of water-quality constituents provides accurate results only for short water-quality time steps. Overly long time steps can yield errors in concentration estimates and can result in situations in which constituent mass is not conserved. The use of a time step that is sufficiently short to avoid these problems may not always be feasible. The absence of EPANET errors or warnings does not ensure conservation of mass. This paper provides examples illustrating mass imbalances and explains how such imbalances can occur because of fundamental limitations in the water-quality routing algorithm used in EPANET. In general, these limitations cannot be overcome by the use of improved waterquality modeling practices. This paper also presents a preliminary event-driven approach that conserves mass with a water-quality time step that is as long as the hydraulic time step. Results obtained using the current approach converge, or tend to converge, toward those obtained using the preliminary event-driven approach as the water-quality time step decreases. Improving the water-quality routing algorithm used in EPANET could eliminate mass imbalances and related errors in estimated concentrations. The results presented in this paper should be of value to those who perform water-quality simulations using EPANET or use the results of such simulations, including utility managers and engineers.
\end{abstract}

Copyright statement. Copyright for this publication of authors Michael J. Davis and Thomas N. Taxon is transferred to UChicago Argonne, LLC, as operator of Argonne National Laboratory, and is subject to reserved government use rights.

\section{Introduction}

EPANET (Rossman, 2000; US EPA, 2017a) is the standard software used for simulating water quality in a water distribution system (WDS). It has been widely and successfully applied for many years. The software includes a hydraulic model that determines water flow and direction throughout a network model that is used to represent a WDS. The network model consists of links (pipes) and nodes (junctions). The water-quality simulation is piggybacked on the hydraulic simulation. EPANET has commonly been used in situations in which water quality does not change rapidly during the simulation. However, in some cases involving simulations of contaminant injections into a WDS it has been found that the mass of the constituent added to the network is not conserved (Davis and Janke, 2014; Davis et al., 2016). That is, at a time $t$ in a simulation, the mass of the constituent in the network's pipes, $M_{\mathrm{P}}(t)$, and tanks, $M_{\mathrm{T}}(t)$, plus the cumulative mass of the constituent removed from the network by nodal demands, $M_{\mathrm{CR}}(t)$, does not equal the cumulative mass of the constituent injected into the network, $M_{\mathrm{CI}}(t)$. (The mass of the constituent in the system before the injection is zero and there is no loss of the constituent due to chemical reactions.) The mass imbalance can be large. For example, defining a massbalance ratio $(\mathrm{MBR})$ as $\left(M_{\mathrm{P}}(t)+M_{\mathrm{T}}(t)+M_{\mathrm{CR}}(t)\right) / M_{\mathrm{CI}}(t)$, the MBR can exceed 10 or be less than 0.1 in some cases for some network models at the end of a simulation. There 
can be cases in which constituent mass is gained during a simulation $(\mathrm{MBR}>1)$, cases in which constituent mass is lost $(\mathrm{MBR}<1)$, as well as cases in which mass is conserved $(\mathrm{MBR}=1)$. A failure to conserve constituent mass indicates that there are errors in the estimated constituent concentrations, which potentially could be a concern for any application that considers water quality in a distribution system. When poor-quality network models are used, the lack of conservation of constituent mass can be exacerbated.

EPANET is available in two forms: (1) a Microsoft Windows $^{\circledR}$ version with a user interface and (2) a programmer's toolkit version. The latter consists of a dynamic link library of functions that allows software developers to customize their EPANET applications. The last major release of EPANET, including both versions, occurred in 2000 (EPANET 2.0). The last minor release of EPANET occurred in 2008 (2.00.12). In 2012, the US EPA initiated a collaborative, community-based open-source effort for EPANET, the "Water Distribution Network Model" project (US EPA, 2017b). In June 2015, an independent water-communityorganized, open-source project began (OpenWaterAnalytics, 2017a); an open-source-project version of the EPANET programmer's toolkit (2.1) was produced in July 2016. Also in July 2016, Lewis Rossman, the original developer of EPANET, contributed a development version (EPANET 3) of the programmer's toolkit (OpenWaterAnalytics, 2017b), which can provide mass-balance information in a status report after a water-quality simulation. None of the earlier versions of the software provides this information.

Although versions 2.00.12 and 2.1 of EPANET do not track the mass of a water-quality constituent and its location in a network during a simulation, both mass and its location can be determined using the concentrations of the constituent provided by the water-quality simulation. EPANET Example Network 3 (US EPA, 2017a) is a simple network with 97 nodes; it is called Network N1 in this paper. Considering independent contaminant injections at each of the nodes in the network, Fig. 1 shows how MBRs determined for these injections are distributed after a $24 \mathrm{~h}$ simulation. (Details on the method used to obtain these results are provided below.) The figure shows that sizable imbalances can occur in this network, unless quite small water-quality time steps are used. (EPANET's default water-quality time step is 300 s.) These imbalances are the result of errors in the concentrations of the constituent determined by EPANET. The water-quality routing algorithm used in EPANET does not ensure conservation of mass and large imbalances can occur because of fundamental limitations in the algorithm. Good modeling practice requires use of a water-quality time step that is less than or equal to one-tenth of the hydraulic time step, which was $3600 \mathrm{~s}$ for the example shown in Fig. 1. Although mass imbalances for all injection nodes for Network N1 can be minimized, but not eliminated, by using a waterquality time step of $60 \mathrm{~s}$ (one-sixtieth of the hydraulic time step), simply reducing the time step will not, in general, en-

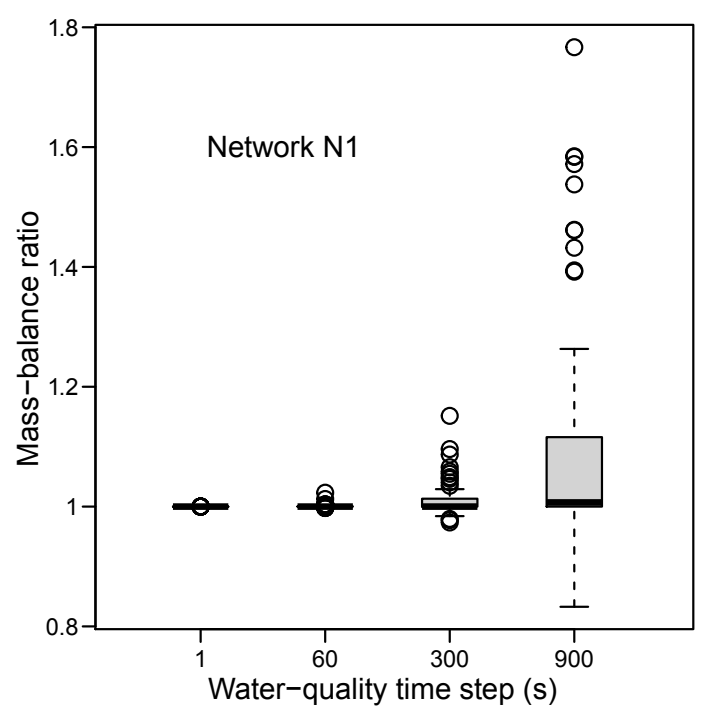

Figure 1. Distribution of mass-balance ratios for EPANET Example Network 3 after $24 \mathrm{~h}$ simulations of independent contaminant injections at each network node. The horizontal black lines in the box plots give the median, the box extends from the lower to the upper quartile, and the whiskers extend to the smaller of 1.5 times the interquartile range or the most extreme data point. The hydraulic time step was $3600 \mathrm{~s}$. $N=94$. Three nodes were excluded because there was no flow at the time the injection occurred.

sure mass conservation. In general, mass conservation cannot be ensured with the use of a nonzero water-quality time step.

This paper provides examples in which mass imbalances occur, discusses why they occur, and presents a preliminary approach to water-quality modeling, currently under development for use in EPANET, which can eliminate such imbalances. Both the approach currently used in EPANET and the preliminary approach included in this paper use Lagrangian water-quality models: they follow individual parcels of water as they move through the network. However, the current EPANET approach uses a time-driven simulation model, while the preliminary approach presented here uses an eventdriven one. The nature of time-driven and event-driven models is discussed later in this paper.

The next section discusses the methods used in our analysis. The nature of the problems encountered when using the current version of EPANET is then described, followed by a section that (1) presents an event-driven simulation method for water-quality routing for EPANET that eliminates these problems and (2) compares results obtained using the two methods. Finally, the major conclusions of the paper are presented, followed by some recommendations. Details on the time-driven and event-driven models are presented in appendices. Although the term "contaminant" is often used in this paper to refer to a water-quality constituent intentionally added to the water in a WDS, the results presented here apply to any water-quality constituent present in a WDS. 


\section{Methods}

The analysis for this paper was done with TEVA-SPOT (US EPA, 2017c), which uses a modified version of the EPANET programmer's toolkit (2.00.12) for hydraulic and waterquality simulations in a WDS (US EPA, 2017a). The version of TEVA-SPOT used was TEVA-SPOTInstaller-2.3.2MSXb-20170110-DEV. TEVA-SPOT was developed by US EPA's National Homeland Security Research Center to provide an ability to evaluate the consequences of intentional and unintentional releases of a contaminant into a WDS and to design contamination warning systems for a WDS. It is the only program that we are aware of that can be used easily and efficiently to evaluate the consequences of injections at any or all nodes in a network model. The ability to track contaminant mass using the concentration results provided by EPANET was included in TEVA-SPOT to allow a better understanding of the distribution of a contaminant in a network following injection and to improve quality control for simulations. Without this capability, the failure to conserve constituent mass that can occur during EPANET simulations would not have been identified. The only significant modification made to the EPANET 2.00.12 code to support its use in TEVA-SPOT is the inclusion of the ability to allow the direct addition of contaminant mass to tanks. Any mass imbalances identified are the result of errors in constituent concentrations provided by EPANET, not the accounting done by TEVA-SPOT.

Water-quality simulations were carried out with the timedriven water-quality model included in EPANET using four network models. Independent injection of a contaminant was simulated at all nodes in a network model (using the MASS source type in EPANET) and concentrations were determined at all downstream nodes for a $168 \mathrm{~h}$ simulation (unless noted otherwise). All simulations used $0.5 \mathrm{~kg}$ of contaminant injected uniformly at a rate of $8.33 \mathrm{~g} \mathrm{~min}^{-1}$ over the period from 00:00 to 01:00 local time (LT), at the beginning of the simulation (again, unless noted otherwise). In addition, the contaminant mass in pipes and tanks and the cumulative mass of contaminant withdrawn from the network were determined at each reporting step in the simulation and MBRs were calculated. Contaminants were assumed to behave as conservative tracers, with concentrations averaged over reporting intervals. Statistics on mass imbalances were determined for each network and specific injection nodes for which notable imbalances were observed were selected for evaluation of contaminant concentrations at downstream nodes. For comparison, simulations also were done for the selected injection nodes using the preliminary event-driven simulation model described here, with the same injection scenario as used with the time-driven method. The time and duration used for injections are arbitrary; however, a consistent injection scenario is necessary to ensure consistent hydraulic conditions for water-quality simulations.
Table 1. Network descriptions.

\begin{tabular}{lrrrr}
\hline & \multicolumn{4}{c}{ Network } \\
\cline { 2 - 5 } Quantity & $\mathrm{N} 1$ & $\mathrm{~N} 2$ & $\mathrm{~N} 3$ & $\mathrm{~N} 4$ \\
\hline Population $\left(10^{3}\right)$ & 79 & 5 & 130 & 250 \\
Mean water use $\left(\mathrm{m}^{3} \mathrm{~s}^{-1}\right)$ & 0.7 & 0.07 & 0.4 & 1.4 \\
Per capita use $\left(\mathrm{Ld}^{-1}\right)$ & 760 & 1200 & 280 & 480 \\
Nodes $\left(10^{3}\right)$ & 0.097 & 1.6 & 6.8 & 13 \\
NZD nodes $\left(10^{3}\right)$ & 0.059 & 0.69 & 6.7 & 11 \\
Pipes $\left(10^{3}\right)$ & 0.12 & 1.4 & 8.0 & 15 \\
Tanks & 3 & 1 & 5 & 2 \\
Reservoirs & 2 & 2 & 1 & 2 \\
Pumps & 2 & 8 & 20 & 4 \\
Valves & 0 & 200 & 16 & 5 \\
\hline
\end{tabular}

Note that all numbers are rounded independently to two significant figures. NZD: nonzero demand.

Except as noted, all simulations used a hydraulic time step of $3600 \mathrm{~s}$. Various water-quality time steps were used for time-driven simulations to determine the influence of the time step on the MBR and the constituent concentrations. The default water-quality time step in EPANET is $300 \mathrm{~s}$, as noted above; however, some studies, e.g., Diao et al. (2016), Helbling and VanBriesen (2009), and Wang and Harrison (2014), use substantially longer time steps. Therefore, in our analysis we include water-quality time steps longer than the default value. A reporting time step of $3600 \mathrm{~s}$ was used in all simulations. Event-driven simulations used a water-quality time step of $3600 \mathrm{~s}$. A quality tolerance of $0.01 \mathrm{mg} \mathrm{L}^{-1}$ was used for all simulations, except as noted.

The network models used are summarized in Table 1. Network N1 is EPANET Example Network 3 (US EPA, 2017a). Network N2 is a synthetic network called Micropolis (Brumbelow et al., 2007). Network N3 is a model for an actual distribution system that has been used in previous studies, e.g., (Davis et al., 2014). Finally, Network N4 is Network 2 in the paper "The battle of the water sensor networks (BWSN)" by (Ostfeld et al., 2008). The version of Network N4 used in this study is available; see the section below on code and data availability. No warnings or errors occurred while using EPANET with the network models and cases considered in this paper. Network schematics are provided in Appendix A.

\section{Simulations with EPANET's time-driven approach}

The time-driven approach used in EPANET is discussed and examples are provided of cases in which the approach does not conserve constituent mass.

\subsection{Background}

EPANET uses the Lagrangian time-driven simulation method for water-quality routing in a network discussed in (Rossman and Boulos, 1996). In general, the method may 
not always provide exact results. In particular, if the waterquality time step is too long, concentration errors can occur; time steps should be less than the time required for a water parcel to move through the network pipe segment (link) having the shortest travel time for the simulation. In principle, errors can be avoided if a sufficiently short time step is used. However, such time steps may not be practical or feasible from a computational perspective. For example, pumps and valves have zero length in EPANET. Also, EPANET allows a minimum time step of only $1 \mathrm{~s}$, which in some cases may not be sufficiently short. Finally, water parcels can move through only one link in a water-quality time step. Although relevant improvements, for example the use of parallel processing and graphics processing units (Wu, 2014), can be expected, the approach currently used in EPANET can be, and will likely remain, computationally challenging because of the possibility of a large number of links in a network and the need to use a short time step to minimize concentration errors.

The algorithm used in EPANET to route water quality through a network can result in situations in which constituent mass is gained or lost. These imbalances can occur because of the manner in which water volume and constituent mass are accumulated at nodes and the manner in which volume and concentration are determined for subsequent releases to downstream links. Constituent mass can be generated during the accumulation step and lost during the release step at locations for which the volume of water being moved during a water-quality time step exceeds the volume of the link in which the water is being moved. When there is a spatial gradient in constituent concentration at such locations, the mass generated during the accumulation step and lost during the release step will not be the same and a net generation or loss of constituent mass can occur. A detailed example is provided in Appendix B illustrating how the timedriven algorithm used in EPANET can fail to conserve constituent mass in such situations.

Restricting the movement of water parcels to only one link per water-quality time step means that, when longer time steps are used, a longer time is required for a parcel to reach a particular downstream location. For example, the arrival time of a contaminant pulse at some location following an upstream injection will be delayed if a longer time step is used. This results in concentration errors even if the shape of the pulse is unaffected. If delays are sufficiently long, the potential exists for changes in hydraulic conditions, which could also affect concentrations. Errors in concentrations due to the effects associated with allowing water parcels to move through only one link per water-quality time step can occur even if mass is conserved.

\subsection{Examples illustrating mass imbalances}

The examples presented in this section were obtained using EPANET's time-driven water-quality routing algorithm; they demonstrate that large mass imbalances can occur, that mass-balance and concentration results can be sensitive to the water-quality time step used, and that a very short time step may be necessary to avoid significant mass imbalances and to minimize concentration errors. These results indicate that to model contaminant intrusion events more accurately a more robust algorithm is needed for use with EPANET that can ensure conservation of mass during water-quality simulations.

For a contaminant injection at a selected node in Network N3, Fig. 2 shows how the various components of massbalance change as the water-quality time step is varied using the time-driven simulation method in EPANET. Note that different vertical scales are used in each plot in the figure. The mass that is injected or removed is a cumulative mass; the mass in pipes and tanks is the mass in those locations at each time in the simulation. For an injection at Node 100 in Network N3, a significant mass imbalance can occur for waterquality time steps of $60 \mathrm{~s}$ or longer. At the end of the $168 \mathrm{~h}$ simulations, the MBRs are 7.11, 4.88, 1.38, and 1 for waterquality time steps of $900,300,60$, and $1 \mathrm{~s}$, respectively. For the time steps equal to or longer than $60 \mathrm{~s}$, considerably more mass was removed from the network than was injected. A time step less than $60 \mathrm{~s}$ is necessary to conserve mass in this case.

Changes in MBRs following injections at Node IN1029 in Network N2 (Micropolis) are shown in Fig. 3 for the timedriven simulation method and four different water-quality time steps. Considerable time is required before the ratios stabilize for the longer time steps. Only for a time step of $1 \mathrm{~s}$ does the MBR approximately equal 1.0. This network contains 196 valves, which, as noted, have zero length in EPANET, and, therefore, zero travel time, which likely contributes to the large MBR values shown in the figure. Note in Fig. 3 that the mass imbalances are larger for a time step of $300 \mathrm{~s}$ than for a time step of $900 \mathrm{~s}$. The location of Node IN1029 is shown in Fig. A3.

Using two different water-quality time steps, Fig. 4 shows how the MBR varies during simulations for an injection at Node JUNCTION-3064 in Network N4 (BWSN), again using the time-driven method. Although the ratio stabilizes after about $20 \mathrm{~h}$ at 1.006 for a time step of $300 \mathrm{~s}$ and at about 1.000 for a time step of $60 \mathrm{~s}$, the ratio can be significantly different from 1.0 during the early portion of the simulations, even with a water-quality time step of $60 \mathrm{~s}$. Mass is first lost from the system, then gained, then lost again, before the ratio approximately stabilizes. Note that the vertical scales on the two plots in Fig. 4 are different.

Figure 5 compares contaminant concentrations obtained using the time-driven method for Node 247 in Network N1 (EPANET Example Network 3) following an injection at Node 101, for different water-quality time steps. As the water-quality time step decreases, the magnitude and timing of the contaminant pulses at the downstream node change. The concentrations appear to stabilize when the time step is 
N3: injection at 00:00 LT at Node 100

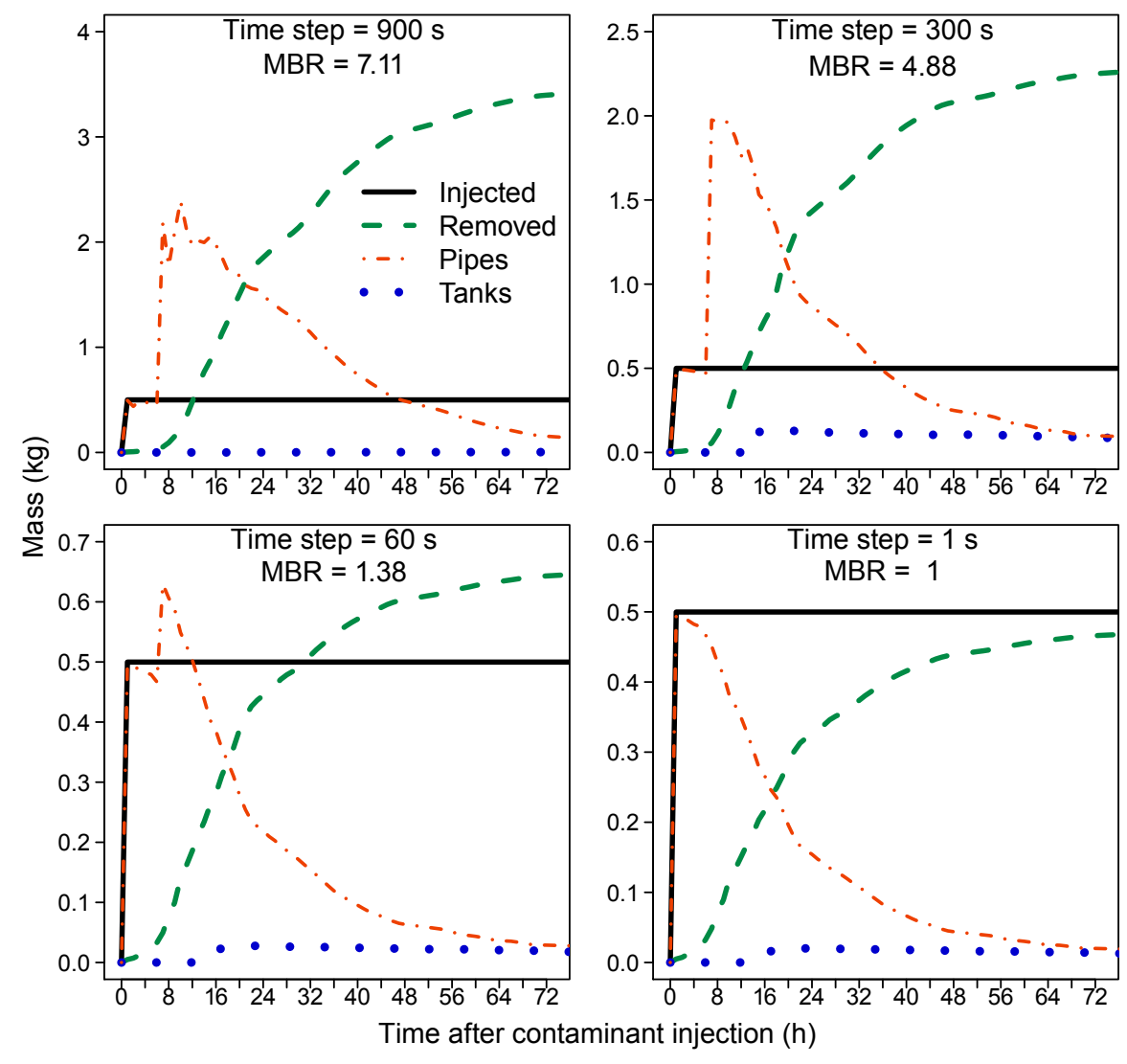

Figure 2. Influence of the water-quality time step on the components of mass balance for an injection at Node 100 in Network N3.

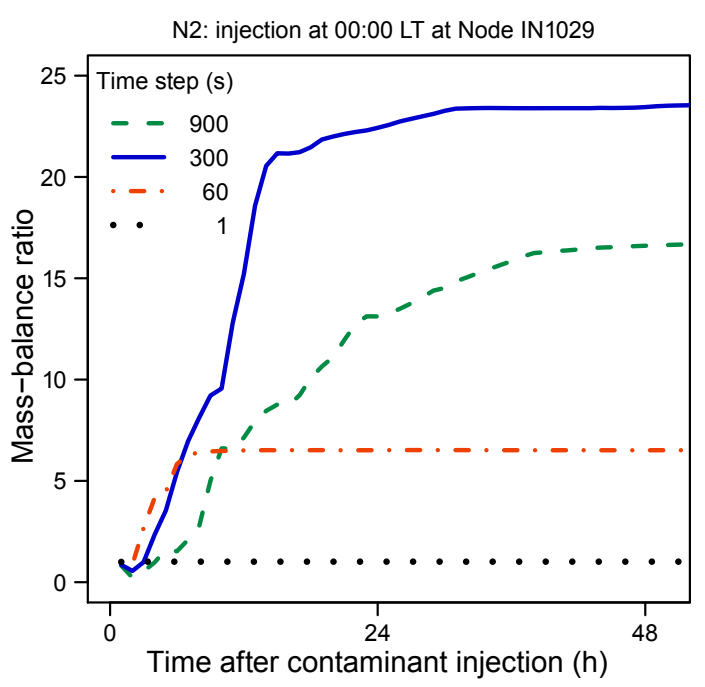

Figure 3. Mass-balance ratios for injections at Node IN1029 in Network N2 during simulations with different water-quality time steps. The location of Node IN1029 is shown in Fig. A3. reduced to $60 \mathrm{~s}$. The MBRs given in the figure are values at the end of a $24 \mathrm{~h}$ simulation.

Contaminant concentrations at Node TN1810 in Network N2 following an injection at Node IN1029 were determined using the time-driven method and are compared in Fig. 6 for different water-quality time steps. Again, the magnitude and timing of the contaminant pulses vary with the time step used. However, in this case, rather than generally decreasing as the time step decreases, the concentration of the pulse increases substantially in magnitude as the time step is reduced from 900 to $300 \mathrm{~s}$, consistent with the MBRs determined for this injection node and shown in Fig. 3. The maximum concentration then decreases by over $99 \%$ going from a time step of $300 \mathrm{~s}$ to a time step of $1 \mathrm{~s}$. The MBRs given in the figure are values at the end of a $168 \mathrm{~h}$ simulation.

Using the time-driven method, contaminant concentrations were obtained at Node 200 in Network N3 after an injection at Node 100; they are compared in Fig. 7 for different water-quality time steps. Again, the timing and magnitude of the contaminant pulse change as the time step is reduced to $1 \mathrm{~s}$, as is the case in the examples presented for Networks N1 and N2. The MBRs given in the figure are values at the end 


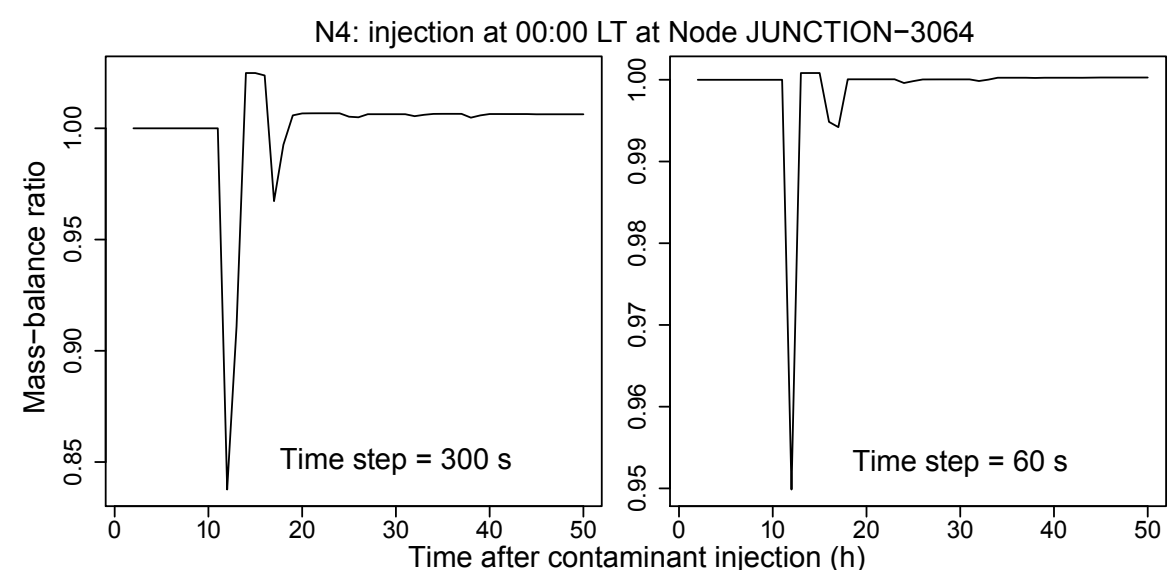

Figure 4. Examples of how the mass-balance ratio can vary during simulations. Results are for an injection at Node JUNCTION-3064 in Network N4. The location of Node JUNCTION-3064 is shown in Fig. A4.

of a $168 \mathrm{~h}$ simulation. Figure 2 shows how the components of mass balance vary following an injection at Node 100 for the same time steps used in Fig. 7.

Figure 8 compares contaminant concentrations obtained for a receptor node, TANK-12525, in Network N4 following an injection at Node JUNCTION-2514, using different water-quality time steps. Again, the simulations used the time-driven method. In this case, the timing of changes in concentration is similar for all the time steps used and there is a high degree of correlation between the results for the different time steps. However, concentration increases consistently as the time step decreases, unlike the trends in the previous examples. Concentrations appear to have approximately stabilized by a time step of $60 \mathrm{~s}$, with little increase in concentrations occurring when the time step is reduced to $1 \mathrm{~s}$. The MBRs given in the figure were determined at the end of a $168 \mathrm{~h}$ simulation. Note that the MBR is less than 1.0 for time steps greater than $1 \mathrm{~s}$. Substantial losses of contaminant mass can occur for longer time steps.

Statistics on the extent of mass imbalance at the end of simulations that used the time-driven method are provided in Table 2 for the four networks considered. The results in the table are based on independent injections at most nodes for each of the networks. For example, the statistics for Network $\mathrm{N} 4$ are based on independent simulations of injections done for most of the 12523 nodes in the network; a small fraction of the nodes were excluded, as discussed in the next paragraph. The table provides the range in MBRs determined for each network for each of four water-quality time steps and the fraction of injection nodes for which there were imbalances above some thresholds (e.g., 1, 5, 10\%).

MBRs equal to zero were obtained for injections at some nodes: the numerator in the MBR was zero because no mass was present in the pipes or tanks at the end of the simulation and no mass was removed during the simulation. Such cases can occur when there is no flow at the time of injec- tion (e.g., zero-demand nodes). When there is no flow at the time of injection, no contaminant mass is added to the water in the WDS and the injected mass is effectively lost, although the accounting process considers it to be mass injected when determining an MBR. Injection nodes for which an MBR was equal to zero at the end of a simulation were not included when determining the statistics shown in Table 2. For Network N4, two additional nodes (JUNCTION9097 and JUNCTION-12348) also were excluded. These two nodes are in dead-end areas with no demands. Therefore, there should be no flows in these areas. However, the network model had a small initial flow at these nodes, inconsistent with a lack of demands in the dead-end areas. (EPANET determines concentrations of constituents in outflows from a node using the flow-weighted sum of inflow concentrations. If other inflows are very small, such anomalous flows could be significant in relative terms and result in concentration anomalies as well.) For Network N3, five nodes had MBRs near 0.2 for all water-quality time steps; however, when the hydraulic and reporting time steps were changed to $1 \mathrm{~min}$, the MBRs for the $1 \mathrm{~min}$ and $1 \mathrm{~s}$ water-quality time steps were near 1 . MBRs near 1 were used for the five nodes when determining the statistics for Network N3 shown in Table 2.

Table 2 shows that as the water-quality time step decreases, the maximum MBRs for each network decrease towards 1.0 and the minimum MBRs generally increase. However, for Network N2 the minimum MBR increased only to 0.08 for the $1 \mathrm{~s}$ time step and for Network N4 it reached only 0.83 . For all four networks considered, the fraction of injection nodes with imbalances above the thresholds listed in the table decreases consistently as the time step decreases. For a time step of $1 \mathrm{~s}$, only about 2,1 , and $<1 \%$ of the nodes had imbalances greater than $1 \%$ for Networks $\mathrm{N} 2, \mathrm{~N} 3$, and $\mathrm{N} 4$, respectively. There were no mass imbalances greater than $0.01 \%$ for this time step for Network N1 (excluding three nodes for which the MBR was zero). This is in contrast to 
Table 2. Statistics for mass imbalances.

\begin{tabular}{lrrr|rrrr}
\hline & & \multicolumn{2}{c}{ MBR } & \multicolumn{3}{c}{ Injection nodes (\%) with imbalances } \\
\cline { 3 - 8 } Network & WQTS (s) & Max. & Min. & $>1 \%$ & $>5 \%$ & $>10 \%$ & $>50 \%$ \\
\hline N1 & 1 & 1.00 & 1.00 & 0 & 0 & 0 & 0 \\
& 60 & 1.02 & $>0.99$ & 2 & 0 & 0 & 0 \\
& 300 & 1.15 & 0.97 & 38 & 6 & 1 & 0 \\
N2 & 900 & 1.77 & 0.83 & 65 & 46 & 31 & 5 \\
& 1 & 1.05 & 0.08 & 2 & 1 & 1 & 1 \\
& 60 & 6.49 & 0.06 & 39 & 29 & 18 & 5 \\
& 300 & 23.45 & $<0.01$ & 47 & 39 & 32 & 17 \\
N3 & 900 & 31.16 & $<0.01$ & 52 & 49 & 44 & 24 \\
& 1 & 1.03 & 0.99 & 1 & 0 & 0 & 0 \\
& 60 & 1.39 & 0.92 & 7 & 1 & $\mathrm{a}$ & 0 \\
& 300 & 4.88 & 0.22 & 35 & 15 & 8 & 1 \\
$\mathrm{~N} 4$ & 900 & 15.74 & 0.08 & 57 & 36 & 25 & 6 \\
& 1 & 1.01 & 0.83 & $\mathrm{~b}$ & $\mathrm{c}$ & $\mathrm{d}$ & 0 \\
& 60 & 1.04 & 0.67 & 1 & $\mathrm{e}$ & $\mathrm{c}$ & 0 \\
& 300 & 1.21 & 0.61 & 21 & 4 & 2 & 0 \\
& 900 & 2.60 & 0.09 & 44 & 28 & 19 & 1
\end{tabular}

WQTS: water-quality time step; MBR: mass-balance ratio. Results do not include cases with MBR $=0$, which occurs for nodes with no flow at the time of injection. In addition, for Network N4, two nodes also are excluded that are in dead-end areas with no demands, but that have small flows at the beginning of the simulation. A total of three nodes are excluded for Network N1 (3\%), 92 or 93 for Network N2 (6\%), depending on the time step, 55 or 56 for Network N3 (1\%), again depending on the time step, and 404 for network N4 (3\%). See text for discussion. ${ }^{\mathrm{a}} 13$ nodes. ${ }^{\mathrm{b}} 11$ nodes. ${ }^{\mathrm{c}} 2$ nodes. ${ }^{\mathrm{d}} 1$ node. ${ }^{\mathrm{e}} 3$ nodes.

the sizable fraction of nodes in all the networks that have imbalances for a time step of $300 \mathrm{~s}$, although the imbalances for Networks N1 and N4 are relatively minor for this time step, with only about 1 and $2 \%$ of nodes in these networks, respectively, having an imbalance greater than $10 \%$.

For a small fraction of the injection nodes in Networks $\mathrm{N} 2$, N3, and N4, about $0.8,0.5$, and $0.2 \%$ of all nodes, respectively, the MBR did not change as the water-quality time step decreased or did not change so that the MBR converged toward 1.0. The lack of convergence of the MBR for these nodes had limited influence on the statistics in Table 2; it did influence the values shown for the minimum MBR for Network N2, particularly for a time step of $1 \mathrm{~s}$. The nonconvergence can be the result of several factors, including cases involving nodes in dead-end areas, as noted above. In addition, some cases had flows at the time of injection that were unexpected, given a lack of demands; the flows disappeared for subsequent time steps. Some problems appear to be related to the hydraulic solution; these were eliminated if a short (60 s) hydraulic time step was used.

Mass balances are sensitive to the injection time used. An acceptable mass balance for a particular application for a given injection node does not guarantee an acceptable mass balance if hydraulic conditions are changed. As an example of the extreme changes that can occur when hydraulic conditions change, consider an injection at Node VN1263 in Network N2 (see location in Fig. A3), a $168 \mathrm{~h}$ simulation, and a $900 \mathrm{~s}$ water-quality time step. For a $1 \mathrm{~h}$ injection starting at 06:00, the MBR at the end of the simulation was 467. For a $1 \mathrm{~h}$ injection starting at 00:00, the MBR at the end of the simulation was 0.007 . In the first case, much more mass was removed from the network by nodal demands during the simulation than was injected; in the second case, very little mass remained in the system at the end of the simulation or was removed from the system by nodal demands during the simulation. In both cases the extreme values for the MBR are the result of errors in the estimated concentrations downstream from the injection location, too high in the first case and too low in the second. These errors resulted in the erroneous numerical generation or loss of constituent mass in the system.

The cases considered to this point used relatively short, $1 \mathrm{~h}$ injections. However, major mass imbalances also occur for injections with long durations. For example, with a $300 \mathrm{~s}$ water-quality time step, the largest MBR for any injection node in Network $\mathrm{N} 2$ for a $1 \mathrm{~h}$ injection at 00:00 is about 23; for 6 and $12 \mathrm{~h}$ injections at that time the largest MBRs are 1495 and 971, respectively. About 17, 22, and $22 \%$ of injection nodes have mass imbalances greater than $50 \%$ for the 1 , 6 , and $12 \mathrm{~h}$ injections, respectively. About 32, 39, and $35 \%$ have imbalances greater than $10 \%$. The statistics for mass imbalance are relatively insensitive to injection duration for this network. However, mass balances for a particular injection node can be sensitive to the injection duration. For example, for Node VN1263 in Network N2 the MBRs are 3.3, 1495, and 971 for injections at 00:00 with durations of 1,6 , and $12 \mathrm{~h}$, respectively.

EPANET 3, the development version of EPANET (OpenWaterAnalytics, 2017b), also yields results with mass imbal- 


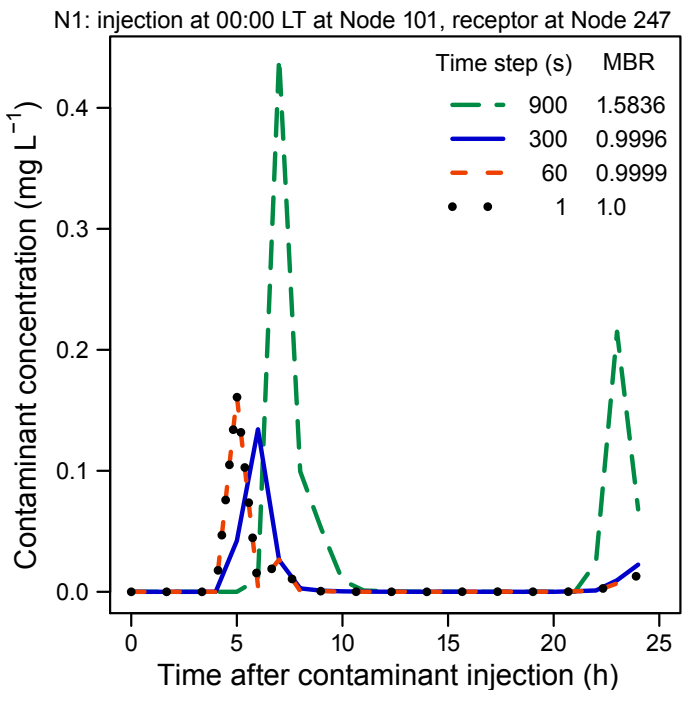

Figure 5. Influence of the water-quality time step on estimated contaminant concentrations at Node 247 in Network N1 following an injection at Node 101 at 00:00 LT. Locations of the nodes are shown in Fig. A1.

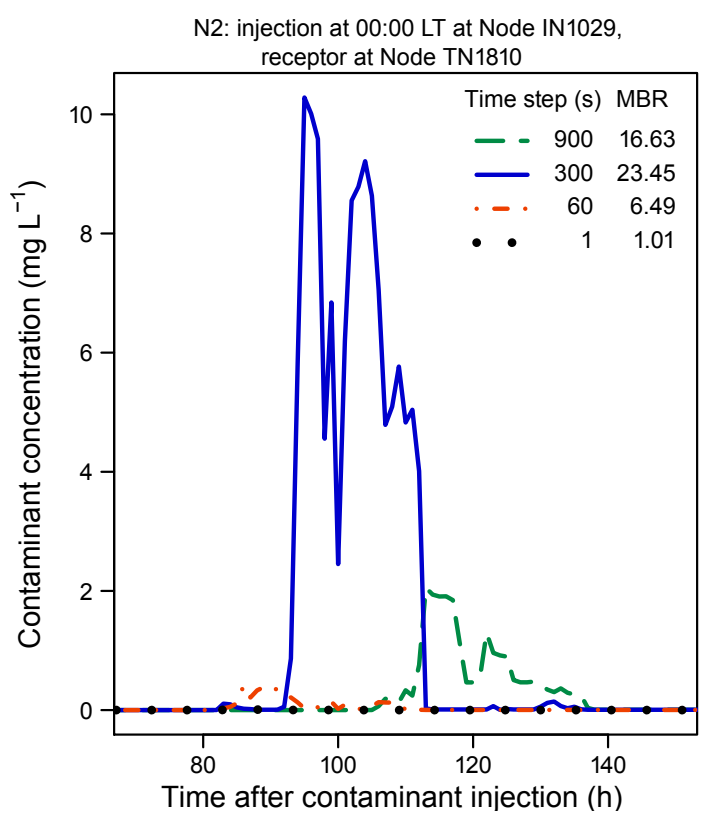

Figure 6. Influence of the water-quality time step on estimated contaminant concentrations at Node TN1810 in Network N2 following an injection at Node IN1029 at 00:00 LT. Locations of the nodes are shown in Fig. A3.

ances. Consider a case involving chlorine decay with both bulk and wall reactions and EPANET Example Network 1 (US EPA, 2017a) with default input parameters, except for the water-quality time step. No water-quality sources were used; however, initial chlorine concentrations were specified. The network is very simple, with only 9 junctions and 12 pipes. A chlorine mass imbalance of $0.85 \%(<1 \%)$ was obtained for a water-quality time step of $900 \mathrm{~s}$ and a $24 \mathrm{~h}$ simulation. For time steps of 300,60 , and $1 \mathrm{~s}$, the imbalances were $0.77,0.58$, and $0.49 \%$, respectively. Mass imbalance was determined in EPANET 3 in the same manner as discussed in this paper except that the initial mass of chlorine in the system also was considered, as was the mass of chlorine lost due to chemical reactions. Execution time increased from about $0.02 \mathrm{~s}$ to about $0.03,0.04$, and $1 \mathrm{~s}$ when the time step was decreased from $900 \mathrm{~s}$ to 300,60 , and $1 \mathrm{~s}$, respectively, an overall increase in execution time of about 50 fold. To obtain mass imbalances well below $0.1 \%$, a time step well below $1 \mathrm{~s}$ may be needed, along with additional increases in execution time. This is an extremely small, simple network and large imbalances are not expected. For this example, the EPANET 3 code (OpenWaterAnalytics, 2017b) was compiled using the GNU Compiler Collection (GCC, 2017).

EPANET allows four source types to be used to define locations of sources of constituents (Rossman, 2000). As noted above, the simulations in this study used the MASS source type, which allows a source strength to be specified in terms of mass added per unit time. The other source types (CONCEN, FLOWPACED, and SETPOINT) all specify source strength in terms of concentration. All are similar in that they allow the addition of the mass of some constituent to the system. EPANET's failure to conserve constituent mass is not related to how the mass is added to the system. Therefore, the findings presented here related to a failure to conserve mass apply independently of the source type used in the simulation. As the example discussed in the preceding paragraph demonstrates, failure to conserve mass can occur in situations in which no sources are used and the only mass present in the system is the result of the initial water quality specified.

\section{Simulations with the preliminary event-driven approach}

The preliminary event-driven approach is discussed and results obtained using this approach are compared to those obtained using the time-driven approach.

\subsection{Background}

Changes in a WDS do not occur at regular time intervals. For example, the time required for a water parcel to move from node to node in the system varies from pipe to pipe and also within a pipe as conditions change. In addition, some pipes can be short, have a high flow rate, and require only a short time for a water parcel to move through them. This transit time can be too small to be practical for use as a water-quality time step in a simulation. A situation in which events (changes) occur at irregular intervals suggests use of an event-driven simulation.

A preliminary event-driven algorithm is outlined here and used to obtain results for comparison with those provided by 
EPANET using the current time-driven approach. The eventdriven approach used is conceptually similar to, but was developed independently of, the Lagrangian event-driven simulation method discussed in (Rossman and Boulos, 1996). An event-driven method updates the state of water quality in the system only when a change (an event) occurs during the simulation, in contrast to the current time-driven method in EPANET, which updates water quality across the entire network at fixed time steps. Numerous events can occur during a time step, as water moves through the network. Various event-driven approaches have been presented previously, for example by Boulos et al. (1994, 1995). The preliminary event-driven algorithm discussed here is included as an option in the current version of TEVA-SPOT (TEVA-SPOTInstaller-2.3.2-MSXb-20170110-DEV) and is being made available to EPANET developers to obtain community support and assistance with improving and evaluating the algorithm.

The event-based, water-quality routing algorithm used here moves homogeneous volumes of water (water parcels with a uniform concentration of a water-quality constituent) through a network. Initially, water parcels are accumulated at all nodes where water enters the system. Nodes with accumulated water parcels from all inflow links are processed in an arbitrary order. Mixing or combining of water parcels occurs at nodes based on the inflow rates of the links flowing into the nodes. Water parcels are combined if the absolute difference between their concentrations is less than some specified amount (the quality tolerance), consistent with the approach used in EPANET 2. After parcels are combined at a node, any nodal demand is removed; the remaining water parcels then are split based on the flow rates of the links flowing from the nodes. These parcels are added to lists of parcels for the downstream links. Any volume in excess of the volume of a link is removed from the leading parcels and placed at the downstream node for further processing. That node is then added to the set of nodes with accumulated water parcels waiting to be processed. Due to recirculating flows, situations can occur in which none of the nodes waiting to be processed has accumulated water parcels on all inflow links. In such cases, an incomplete parcel with the volume that will be moved, but an unspecified concentration, is created for each inflow link that does not have an accumulated inflow. These incomplete parcels are moved, combined, and split in the same manner as parcels for which constituent concentration has been determined; however, internal references are maintained that allow concentrations to be updated when parcels for which concentrations have been determined arrive at a node for which incomplete parcels were created. Flow reversals between hydraulic time steps are accommodated in the same manner as in EPANET 2. The event-driven simulation method provides results that do not depend on the water-quality time step if it is equal to or shorter than the hydraulic time step. The method actually does not require an independent water-quality time step: the simulation is event- driven as long as the hydraulic conditions do not change. Because by construction the method accounts for every individual water parcel, its resulting MBR will always be 1.0. An example illustrating the operation of the algorithm using a case with recirculating flow is provided in Appendix C. Working through the example provided in the appendix will provide a better understanding of the approach outlined in this paragraph.

\subsection{Discussion}

Concentrations obtained using EPANET's time-driven algorithm tend to converge toward those obtained using the event-driven algorithm as the water-quality time step used in the time-driven algorithm decreases. For short water-quality time steps (e.g., $1 \mathrm{~s}$ ) with the time-driven approach, the results for the two methods can be very similar and differences can be difficult to see in the plots used in this paper. Therefore, to better examine this convergence, least-squares fits were determined relating (1) the concentrations obtained using the time-driven approach with a water-quality time step of $1 \mathrm{~s}$ (TD1) and the concentrations obtained using the same approach with a $60 \mathrm{~s}$ time step (TD60), (2) TD1 and the concentrations obtained using the time-driven approach with a $300 \mathrm{~s}$ time step (TD300), and (3) TD1 and the concentrations obtained using the event-driven approach with a $3600 \mathrm{~s}$ time step (ED3600). These least-squares lines have the form $\hat{y}=a x+b$, where $a$ and $b$ are the slope and intercept of the fitted least-squares line, $x$ is the value of TD1, and $\hat{y}$ is the fitted value of TD60, TD300, or ED3600, depending on which is being used. The results of fitting least-squares lines are shown in Table 3 for the four cases examined in Figs. 5 to 8.

The number $(N)$ of hourly concentration values used to obtain the results shown in the Table 3 corresponds approximately to the number of hourly concentration values shown in the figures for the different networks. For Network N1, N was 24 , covering the entire length of the simulation. For Network N2, it was 60 , the length of the middle portion of the plot in Fig. 6. For Network N3, $N$ was 39, the length of the period from hour 1 in the simulation to hour 40 (see Fig. 7). For Network N4, results for the entire $168 \mathrm{~h}$ simulation were used. The water-quality tolerance in the simulations used to obtain the concentrations needed for the analysis presented in the table was 0.01 , except for the event-driven simulations for Network $\mathrm{N} 4$, for which 0.1 was used.

If the concentrations obtained using the time-driven method with a $1 \mathrm{~s}$ water-quality time step are identical to those obtained for one of the other cases, the slope of the least-squares line relating the concentrations will be 1 , the intercept will be 0 , the adjusted $R^{2}$ will be 1 , and the residuals for the fit will all be 0 . From Table 3, the results for Networks $\mathrm{N} 1, \mathrm{~N} 2$, and N3 show that of the three cases considered, the concentrations for TD1 are closest to those for ED3600. Also, the agreement improves going from TD300 to TD60 to ED3600. This improvement is what would be expected if the 
Table 3. Least-squares fits of concentration results.

\begin{tabular}{lllrrrr}
\hline Network & $N$ & Case & $a$ & $b$ & adj- $R^{2}$ & Residual SD \\
\hline $\mathrm{N} 1$ & 24 & TD300 & 0.2547 & 0.0074 & 0.0478 & 0.0273 \\
& & TD60 & 0.9985 & $-1 \times 10^{-6}$ & 0.9999 & 0.0003 \\
& & ED3600 & 0.9995 & 0.00001 & 1 & 0.0001 \\
$\mathrm{~N} 2$ & 60 & TD300 & -24.68 & 2.14 & -0.0169 & 3.385 \\
& & TD60 & 52.42 & 0.0334 & 0.3196 & 0.0911 \\
& & ED3600 & 0.944 & 0.0003 & 0.7657 & 0.0006 \\
$\mathrm{~N} 3$ & 39 & TD300 & 6.159 & -0.0115 & 0.8866 & 0.1398 \\
& & TD60 & 1.515 & -0.00351 & 0.9866 & 0.0112 \\
& & ED3600 & 0.9997 & 0.0001 & 0.9999 & 0.0005 \\
N4 & \multirow{2}{*}{168} & TD300 & 0.7250 & 0.0016 & 0.9956 & 0.0011 \\
& & TD60 & 0.9840 & 0.0001 & 1 & 0.0001 \\
& & ED3600 & 0.9791 & 0.0018 & 0.9762 & 0.0035 \\
\hline
\end{tabular}

This table gives the parameters of a least-squares fit of the concentrations for the cases shown to the concentrations obtained using the time-driven algorithm with a water-quality time step of $1 \mathrm{~s}$. Quantities $a$ and $b$ are the slope and intercept, respectively, of the least-squares line. Cases are TD300, the time-driven algorithm with a $300 \mathrm{~s}$ time step; TD60, the time-driven algorithm with a $60 \mathrm{~s}$ time step; and ED3600, the event-driven algorithm with a $3600 \mathrm{~s}$ time step. $N$ : number of hourly concentration values used. SD: standard deviation.

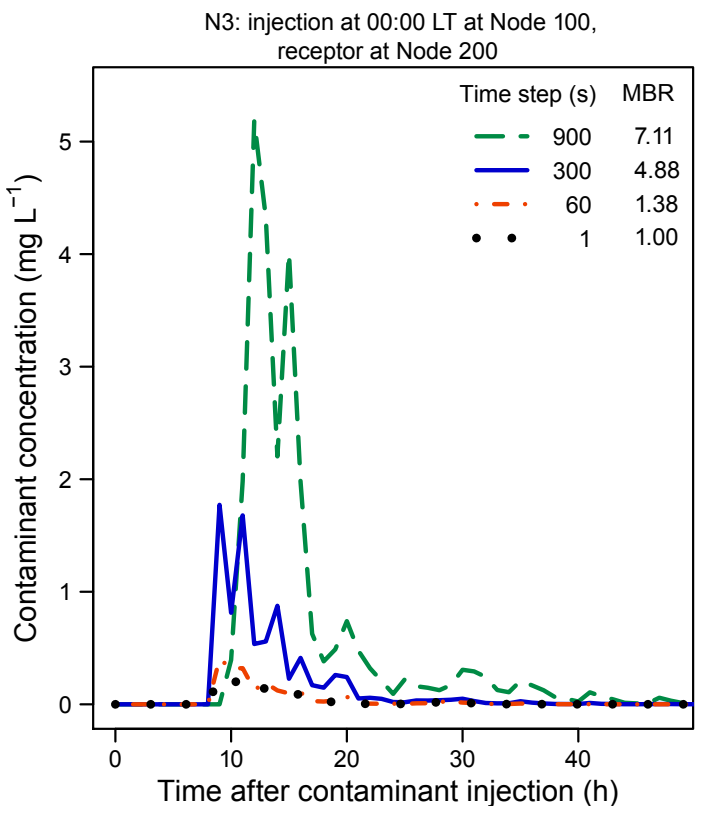

Figure 7. Influence of the water-quality time step on estimated contaminant concentrations at Node 200 in Network N3 following an injection at Node 100 at 00:00 LT.

results for the time-driven approach are converging to those for the event-driven approach as the water-quality time step used for the time-driven approach decreases. For Network $\mathrm{N} 4$ there is a high degree of correlation between the concentrations obtained using the time-driven method with different time steps (cf. Fig. 8) and the quality of the fit is similar for all three cases considered. However, because the magnitude of the concentrations increases as the time step decreases, the

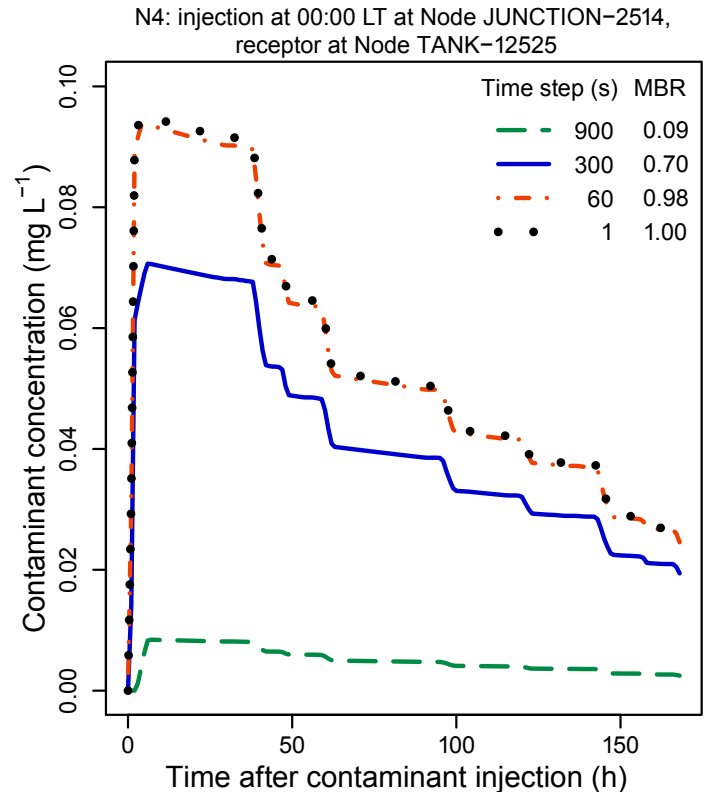

Figure 8. Influence of the water-quality time step on estimated contaminant concentrations at Node TANK-12525 in Network N4 following an injection at Node JUNCTION-2514 at 00:00 LT. Locations of the nodes are shown in Figs. A4 and A5.

slope of the least-squares line increases going from TD300 to TD60.

The results in Table 3 for Network N2 indicate a less-thanperfect correlation between the concentrations obtained using the time-driven algorithm used in EPANET and a waterquality time step of $1 \mathrm{~s}$ and those obtained using the eventdriven algorithm and a time step of $3600 \mathrm{~s}$. The concentrations obtained using the two approaches are compared in 
Table 4. Comparison of execution times for the time-driven and event-driven algorithms.

\begin{tabular}{llrr}
\hline & & \multicolumn{2}{c}{ Execution time (s) } \\
\cline { 3 - 4 } Network & Injection location & TD $^{\mathrm{a}}$ & ED $^{\mathrm{b}}$ \\
\hline N1 & All 97 nodes & 259 & 24 \\
N2 & Node IN1029 & 970 & 294 \\
& Node VN1263 & 326 & 670 \\
N3 & Node 100 & 2990 & 220 \\
& Node 300 & 1440 & 17 \\
N4 & Node JUNCTION-2514 & 1780 & 15 \\
& Node JUNCTION-3064 & 1760 & 17 \\
\hline
\end{tabular}

Note that all simulations were done using a $2.8 \mathrm{GHz}$ processor. The simulation durations were $168 \mathrm{~h}$, except for Network N1, for which they were $24 \mathrm{~h}$. All times are rounded to three significant figures or to the nearest whole second.

TD: time-driven; ED: event-driven. ${ }^{\mathrm{a}}$ Water-quality time step is $1 \mathrm{~s}$.

b Water-quality time step is $3600 \mathrm{~s}^{\mathrm{c}}$ Quality tolerance is $1.0 \mathrm{mg} \mathrm{L}^{-1}$

Fig. 9 (which uses an expanded vertical scale compared to the one used in Fig. 6). The results obtained for the two simulations are noticeably different. However, compared to the differences between the results obtained using the timedriven approach using a $1 \mathrm{~s}$ time step and those obtained using longer time steps, the differences are quite minor, as can be seen from a comparison of Figs. 6 and 9.

Overall, the results presented here demonstrate that substantial mass imbalances can occur during EPANET waterquality simulations. Such mass imbalances tend to disappear and significant changes in constituent concentrations can occur as the water-quality time step becomes small. Also, these constituent concentrations tend to converge toward those obtained using the event-driven simulation method, which conserves constituent mass.

The preliminary event-driven algorithm discussed here currently addresses only those constituents that behave as tracers. The algorithm needs to be expanded to consider constituent decay. The algorithm also needs to be evaluated using a wider range of networks and cases. The accuracy, storage requirements, and computation time for other types of water-quality modeling problems, such as source tracing, water age, and chlorine decay need to be examined. Preliminary results are presented here to help motivate additional efforts to improve water-quality simulations in EPANET.

The results presented here indicate that, in general, a water-quality time step of $1 \mathrm{~s}$ may be necessary to obtain acceptable mass-balance results when using the time-driven approach in EPANET. For large networks, such a time step can require considerable computational effort. Statistics for execution times for TEVA-SPOT, using EPANET and the timedriven algorithm, are provided in (Davis et al., 2016) for several network models, including Network N4 (BWSN, called Network E3 in the reference) for a $1 \mathrm{~s}$ water-quality time step. Results in the reference are for a subset of the nodes considered here, only those with a nonzero demand, and in-

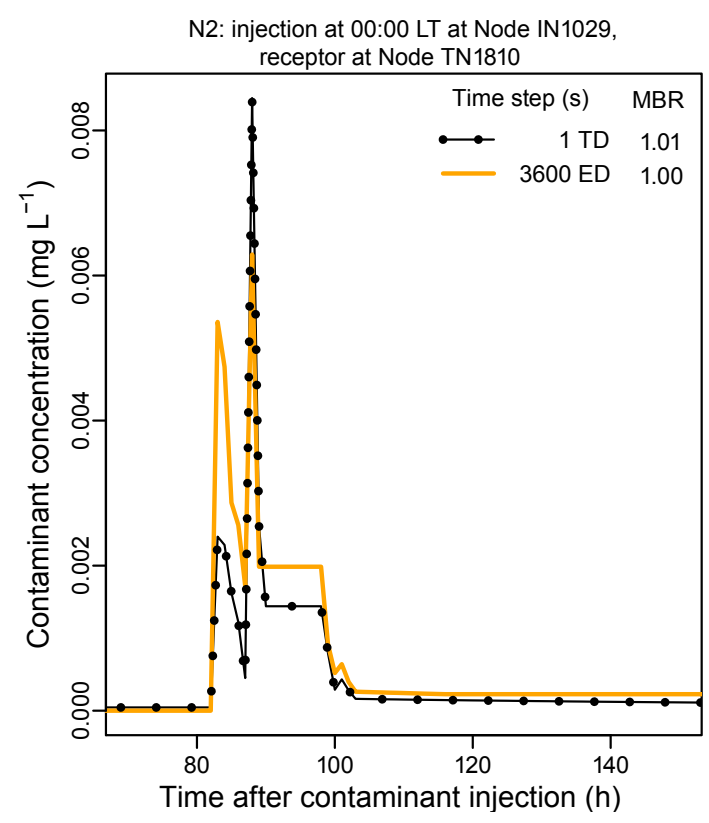

Figure 9. Estimated contaminant concentrations at Node TN1810 in Network N2 following an injection at Node IN1029 obtained using the time-driven (TD) and event-driven (ED) water-quality algorithms. Locations of the nodes are shown in Fig. A3.

clude some additional computations beyond those used for this paper but demonstrate substantial execution times. For a single injection, execution times were about $70 \mathrm{~min}$ using a $2.3 \mathrm{GHz}$ processor. For injections at all nonzero demand nodes for the network, the execution time was about 16 days for a server with four such processors using 32 cores, with 32 simultaneous simulations being performed. The eventdriven algorithm is not fully developed; however, because a water-quality time step as long as the hydraulic time step can be used, it is expected to generally require less computational effort than the time-driven algorithm if conservation of mass is required. Table 4 compares execution times for the time-driven and event-driven algorithms for examples used in this paper. As noted above, these examples were selected to demonstrate mass imbalances. A time step of $1 \mathrm{~s}$ was used for the time-driven algorithm and $3600 \mathrm{~s}$ was used for the event-driven one. To provide a second injection location for Network N3, Node 300 is included, although no example is presented using this node. The execution times were obtained using a single $2.8 \mathrm{GHz}$ processor. In general, for the cases considered here, the event-driven approach requires substantially shorter execution times than the time-driven approach in EPANET when a $1 \mathrm{~s}$ water-quality time step is used.

\section{Conclusions}

As the examples presented here illustrate, the current version of EPANET can produce results for which the mass of a water-quality constituent is not conserved. Significant mass 
imbalances can occur when modeling water quality, even for water-quality time steps considerably shorter than those commonly used with EPANET and that are consistent with good modeling practices. These mass balances are associated with inaccurate estimated constituent concentrations.

Substantial mass imbalances can occur at the beginning of a simulation, but be reduced or eliminated as the simulation proceeds. Therefore, if unacceptable mass imbalances occur for a short simulation, a longer simulation time may be needed.

Although mass imbalances can be reduced or eliminated by decreasing the size of the water-quality time step, sufficient reductions may not be practical. As noted above, the default water-quality time step in EPANET is $300 \mathrm{~s}$ and longer time steps are used in some applications. However, in some cases, as shown here, use of a time step as short as $60 \mathrm{~s} \mathrm{can}$ result in significant errors; a time step less than $60 \mathrm{~s}$ may be necessary to obtain acceptable results and in some cases a time step of $1 \mathrm{~s}$ does not eliminate mass imbalances.

Failure to conserve constituent mass is the result of fundamental limitations in the water-quality routing algorithm used in EPANET. The algorithm does not ensure mass conservation.

Results from the current version of EPANET tend to converge toward those obtained using our preliminary eventdriven water-quality algorithm as the water-quality time step used with the current version of EPANET is reduced. The event-based algorithm for water-quality routing provides results that conserve constituent mass and that are independent of the water-quality time step if it is less than or equal to the hydraulic time step. Given this independence of the size of the water-quality time step, the new algorithm may not only be more accurate, but also more economical to use than the one currently included in EPANET if mass conservation is required.

The event-driven algorithm used here is under development. It is available as an option for water-quality modeling in TEVA-SPOT. Additional refinement of the approach is needed; in particular, it needs to be modified to consider non-conservative constituent behavior.

EPANET water-quality simulations are widely used by water utilities. The results presented here should be of value to utility managers and engineers; they allow users of such simulations to better understand an important potential limitation of these simulations. The results also allow them to understand how these simulations can be improved.

\section{Recommendations}

On the basis of the results presented here, we recommend that the water-quality algorithm used in EPANET be replaced with one that conserves mass and provides accurate concentration estimates. Until such a change can be accomplished, we recommend the following.

1. Capabilities should be added to EPANET to produce reports on the mass balance of water-quality constituents and to provide warning or error statements when conditions are present that could result in a failure to conserve constituent mass or when such a failure actually occurs.

2. When a capability to obtain an evaluation of mass balance is available, the water-quality time step should be selected so that acceptable mass balances are obtained.

3. As long as a time-driven algorithm is used, some value for a default water-quality time step is needed. To reduce opportunities for mass imbalances to occur, the current default value of $300 \mathrm{~s}$ should be reduced.

Code and data availability. Models for Networks N1, N2, and N4 are available at https://doi.org/10.23719/1375314 (US EPA, 2018a). The model for Network N3 is proprietary and cannot be shared. The preliminary, Lagrangian event-driven algorithm discussed in the paper is available for inspection and (hopefully) collaboration at https://github.com/ttaxon/EPANET/ tree/flow-transport-model (Taxon, 2018). The algorithm has been incorporated into TEVA-SPOT and an executable version is available at https://doi.org/10.23719/1375315 (US EPA, 2018b). 


\section{Appendix A: Network maps}

Schematics of Networks N1, N2, and N4 are shown in Figs. A1, A3, and A4, respectively. Additional detail for Network N1 is shown in Fig. A2 and for Network N4 in Fig. A5. Because it contains confidential information, the schematic for Network N3 cannot be provided. The nodes identified in the figures are used in examples discussed in this paper.

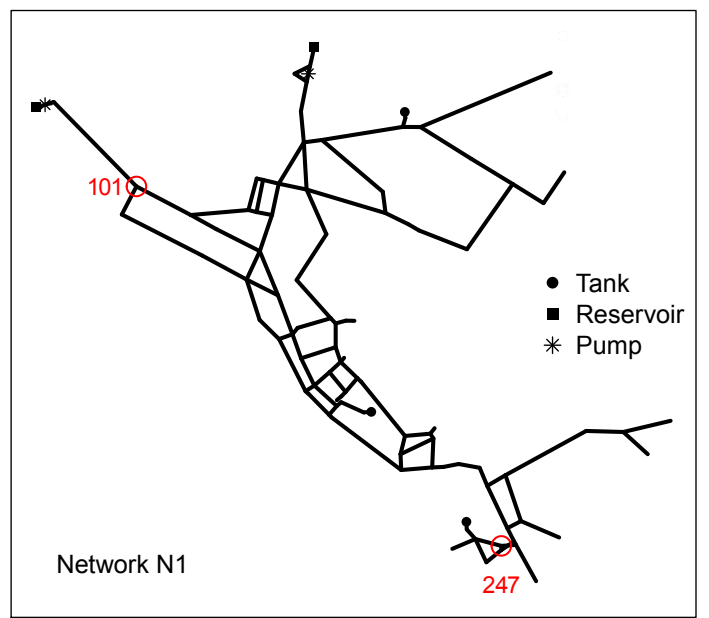

Figure A1. Network N1.

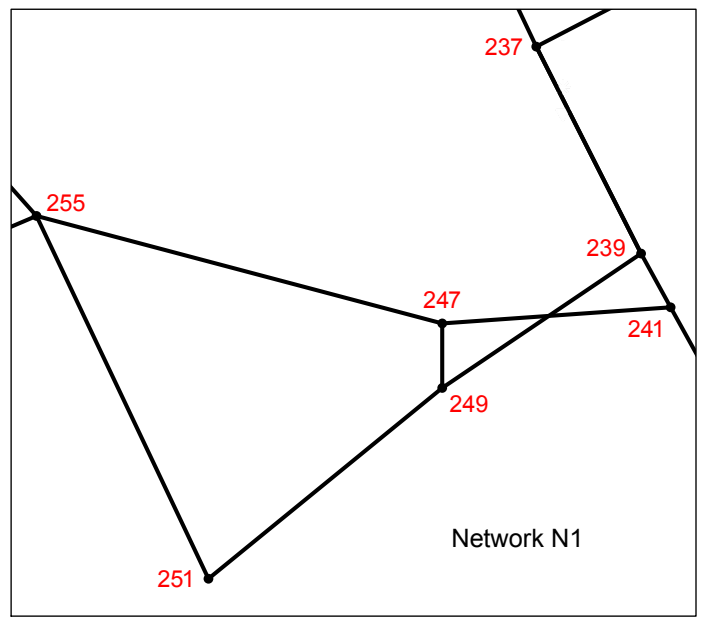

Figure A2. Detail in Network N1.

\section{Appendix B: Example using the time-driven approach}

This appendix presents an example illustrating the operation of the time-driven algorithm used in EPANET and, in particular, shows how the algorithm can fail to conserve constituent mass. The example uses the portion of Network N1 shown in Fig. A2. The example presented here is designed to demonstrate how mass imbalance can occur and is not a suggestion

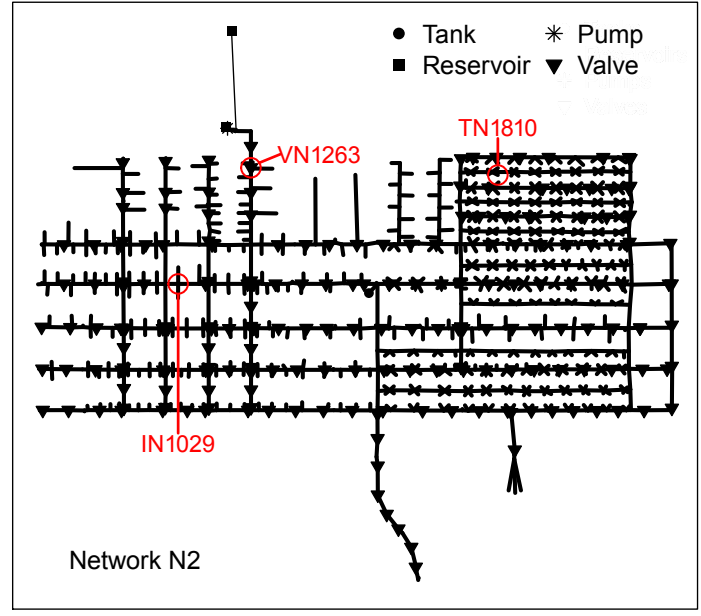

Figure A3. Network N2.

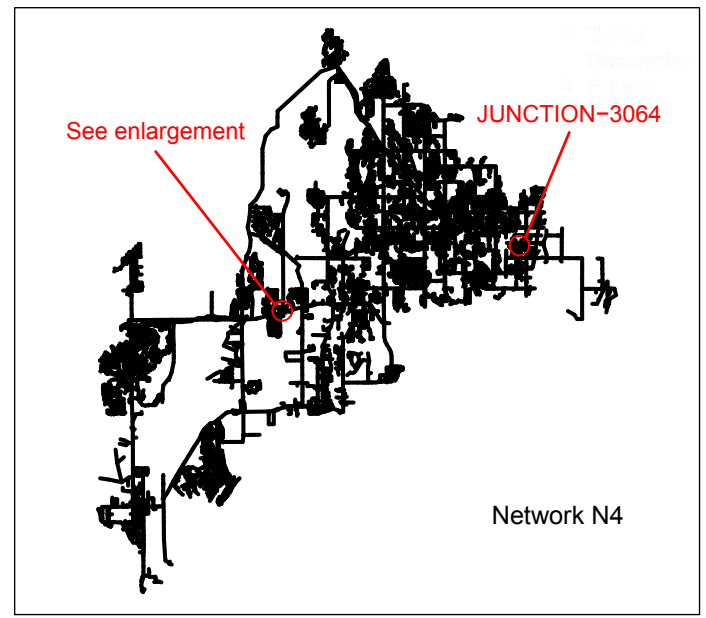

Figure A4. Network N4. See Fig. A5 for an enlargement showing detail in the highlighted area.

that the parameters used in the example would actually be used.

\section{B1 Water-quality routing with the time-driven approach}

Water-quality routing in EPANET uses the function transport(), which in turn uses several other functions to accumulate constituent mass and water volume at nodes and to create new water parcels in outflow links. Accumulating, releasing, and updating are each done at the same time at all nodes in a network (a time-driven approach). The functions listed below are used in the order shown:

- accumulate(). This function accumulates constituent mass (MassIn) and inflow volume (VolIn) at nodes and computes nodal constituent concentrations, which are stored for later use. 


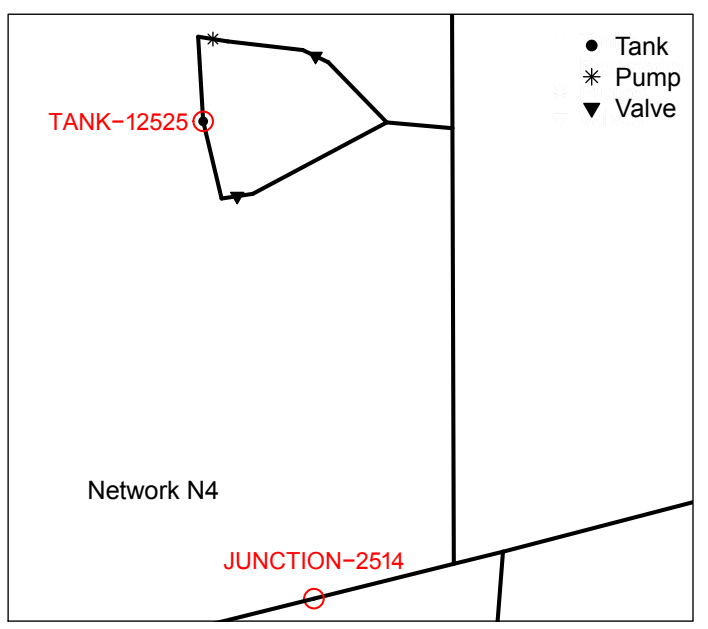

Figure A5. Detail in Network N4.

- updatenodes(). This function updates concentrations at all nodes. It does not consider additions from any sources of water-quality constituents at nodes.

- sourceinput(). If appropriate, this function accounts for any contributions of constituent mass from sources. (No sources are used in the example presented here.)

- release(). This function creates new parcels in outflow links for all nodes.

- updatesourcenodes(). This function updates water quality at source nodes using results from sourceinput() (not relevant for this example).

The next section illustrates the application of the relevant functions using an example based on Network N1.

\section{B2 Example}

For the example presented in this appendix a total of $0.5 \mathrm{~kg}$ of a water-quality constituent was added at a constant rate at Node 249 of Network N1 during the first hour of a $24 \mathrm{~h}$ simulation. Water-quality and hydraulic time steps of $3600 \mathrm{~s}$ were used along with constant nodal demands. The quality tolerance was $0.01 \mathrm{mg} \mathrm{L}^{-1}$.

The initial conditions in the portion of Network N1 (Fig. A2) used in this example are shown in Fig. B1. They correspond to those at hour two in the simulation. The example uses seven nodes $(237,239,241$, and so on). The italicized numbers below or to the right of the links connecting nodes are the link numbers $(273,275$, etc.). There are demands at Nodes 239 and 247. The volumes of water moved during a water-quality time step are shown above or to the left of the links; the arrows indicate the direction of flow for the time step shown. For example, $5.941 \mathrm{~m}^{3}$ of water are moved from Link 281 to Node 247 during the time step and $15.985 \mathrm{~m}^{3}$ of water are removed by demands at
Node 247 during the time step. The volumes of water parcels and the concentrations of a water-quality constituent present in parcels are shown. For example, Link 281 has one water parcel with a volume of $6.873 \mathrm{~m}^{3}$ and a concentration of zero (shown as 6.873/0). Link 283 has two parcels; the leading parcel has a volume of $6.146 \mathrm{~m}^{3}$ and a concentration of $78.084 \mathrm{mg} \mathrm{L}^{-1}(6.146 / 78.084)$ and the trailing parcel has a volume of $3.417 \mathrm{~m}^{3}$ and a concentration of zero $(3.417 / 0)$. The link volume is equal to the sum of volumes of the water parcels in the link; Link 283 has a volume of $6.146+3.417=9.563 \mathrm{~m}^{3}$. The small tables in the figure provide initial values of MassIn, VolIn, and constituent concentrations for Nodes 239, 247, and 249. In these tables the units for MassIn, VolIn, and concentration are $\mathrm{mg}, \mathrm{m}^{3}$, and $\mathrm{mg} \mathrm{L}^{-1}$, respectively.

Conditions after accumulate() has been called are shown in Fig. B2. The volumes and concentrations of parcels remaining after nodal inflows have been removed from links are shown. For some links the volume removed exceeds the volume of the link and no parcels remain in the links. These links are shaded in the figure. When the volume being moved exceeds the volume of a link, the contribution from the link to VolIn for the downstream node is determined using the volume moved. For example, VolIn for Node 239 is $26.998 \mathrm{~m}^{3}$, the volume being moved on Link 273, not the volume of the link, which is $11.343 \mathrm{~m}^{3}$. Values of MassIn for downstream nodes are determined by the volume moved in a time step, not the volume of the water parcels in the links providing inflows. When the volume moved is greater than the link volume, the extra volume that is moved to the downstream node has the same concentration as the trailing water parcel in the link. For example, for Node 247 the constituent mass coming from Link 285 is determined using the volume moved $\left(6.854 \mathrm{~m}^{3}\right)$ and the concentration $\left(78.084 \mathrm{mg} \mathrm{L}^{-1}\right)$ of the water parcel in the link (the only parcel in the link and, therefore, also the trailing parcel), which gives a contribution to MassIn for the node of about $0.535 \mathrm{~kg}$. The concentrations of the water parcels in Links 281 and 287 are zero and the links do not contribute any mass to MassIn for Node 247. The value of MassIn in the table for Node 247 (535 $159 \mathrm{mg}$ ) is slightly different from the product of VolIn and the concentration due to rounding. Note that the value for MassIn for Node $247(0.535 \mathrm{~kg})$ is larger than the constituent mass added to the network $(0.5 \mathrm{~kg})$. Excess mass has been generated at the node; a mass gain of about $0.518 \mathrm{~kg}$ has occurred, given that about $0.535 \mathrm{~kg}$ has been added to MassIn for Node 247 and not $0.017 \mathrm{~kg}$, the mass of constituent in the parcel in Link 285, which has a volume of $0.222 \mathrm{~m}^{3}$ and a concentration of $78.084 \mathrm{mg} \mathrm{L}^{-1}$. Note that the value of VolIn for Node 247 is the sum of the water volumes moved from the three inflow links $(281,285$, and 287).

For Link 283 the link volume is also less than the volume of water being moved in the time step $\left(9.563\right.$ vs. $\left.9.939 \mathrm{~m}^{3}\right)$. The leading water parcel flows into Node 249, contributing a constituent mass of about $0.480 \mathrm{~kg}\left(6.146 \mathrm{~m}^{3}\right.$ multiplied 


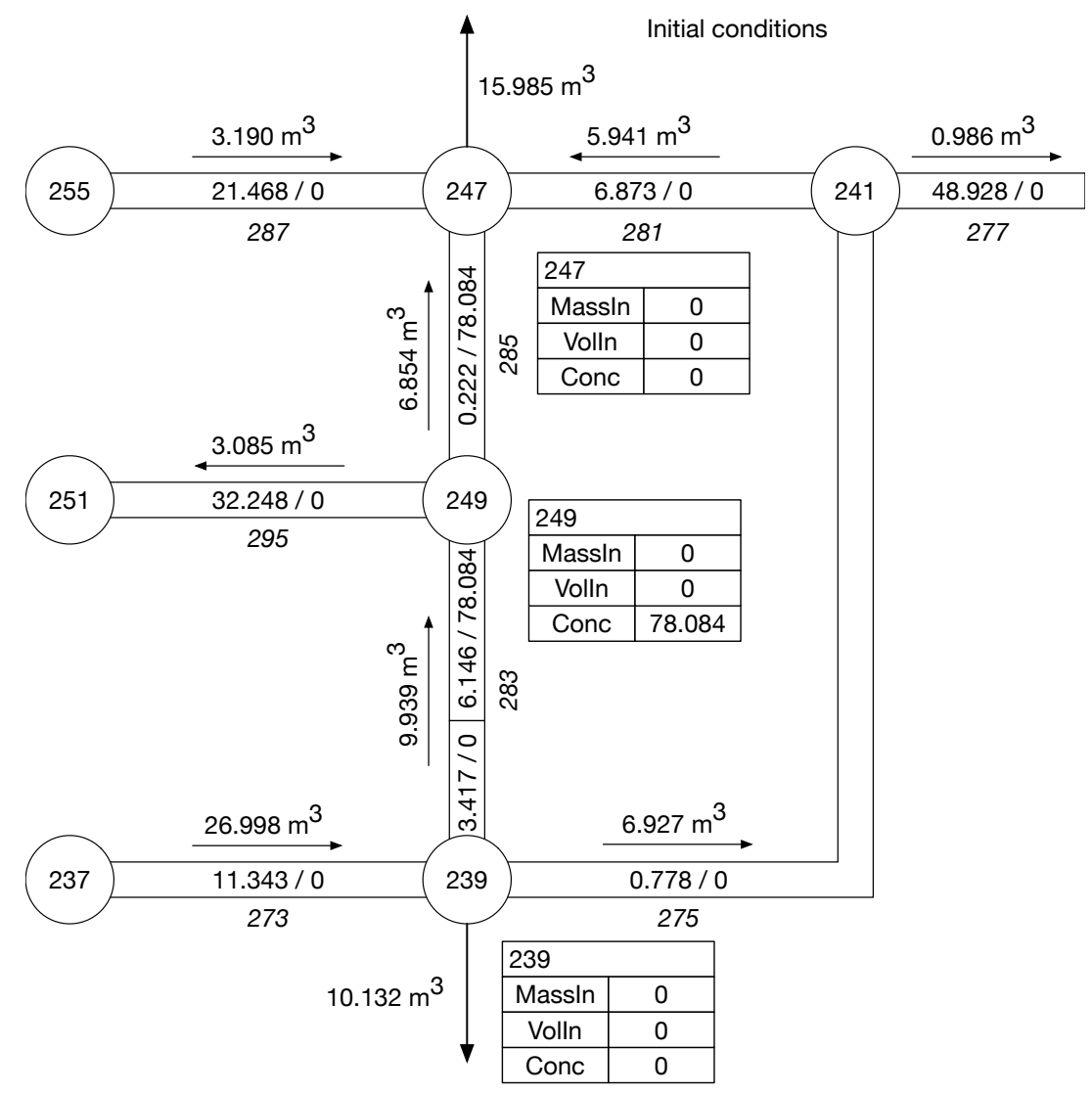

Figure B1. Example: initial conditions.

by $78.084 \mathrm{mg} \mathrm{L}^{-1}$ ). To provide the necessary volume being moved to the node, an extra $0.376 \mathrm{~m}^{3}$ is added to the trailing parcel and given a concentration of zero, the same as concentration of the trailing parcel. The second parcel adds no constituent mass to Node 249; the value for MassIn for the node is about $0.480 \mathrm{~kg}$, coming entirely from the leading water parcel on the link.

No constituent mass is accumulated at Node 239. Although the volume of water being moved in Link 273 is larger than the volume of the link, the concentration of the added volume is zero.

When the accumulate step is completed, values for MassIn and VolIn are computed for all nodes in the network. Values for these quantities are shown in Fig. B2 for Nodes 239, 247, and 249. MassIn is zero for all other nodes. The initial conditions show that the water-quality constituent was present only in Links 283 and 285.

In the next step in the water-quality routing process, updatenodes() is called to update nodal concentrations. The updated concentrations for Nodes 239, 247, and 249 are shown in the tables in Fig. B3. The concentrations are determined using MassIn and VolIn for each node. For example, the con- centration for Node 249 is $48.289 \mathrm{mg} \mathrm{L}^{-1}$, obtained by dividing $479925 \mathrm{mg}$ by $1000 \times 9.939 \mathrm{~m}^{3}$.

In the final step in this example, a call to release() creates new water parcels on the outflow links for all nodes. Conditions after these parcels are created are shown in Fig. B4. New parcels are combined with parcels already present if the difference between their concentrations is less than the quality tolerance $\left(0.01 \mathrm{mg} \mathrm{L}^{-1}\right)$. For example, a new parcel with a volume of $5.941 \mathrm{~m}^{3}$ was added to Link 281 and combined with the $0.931 \mathrm{~m}^{3}$ parcel already on the link because both parcels have a concentration of zero. New parcels with concentrations of $48.289 \mathrm{mg} \mathrm{L}^{-1}$ are added to Links 285 and 295 , using the concentration for Node 249. The volume of the parcel for Link 285 is $0.222 \mathrm{~m}^{3}$, the volume of the link. However, the volume released to the link is $6.854 \mathrm{~m}^{3}$; the difference between this volume and the volume of the link (6.854$0.222=6.632 \mathrm{~m}^{3}$ ) is effectively lost. Because the concentration associated with this volume is $48.289 \mathrm{mg} \mathrm{L}^{-1}$, a mass loss of about $0.320 \mathrm{~kg}(48.289 \times 6.632$ divided by 1000$)$ occurs. Adding the mass gain of $0.518 \mathrm{~kg}$ in the accumulate step, there is a net mass gain at this point in the simulation equal to about $0.2 \mathrm{~kg}$. Given the constituent mass removed by 


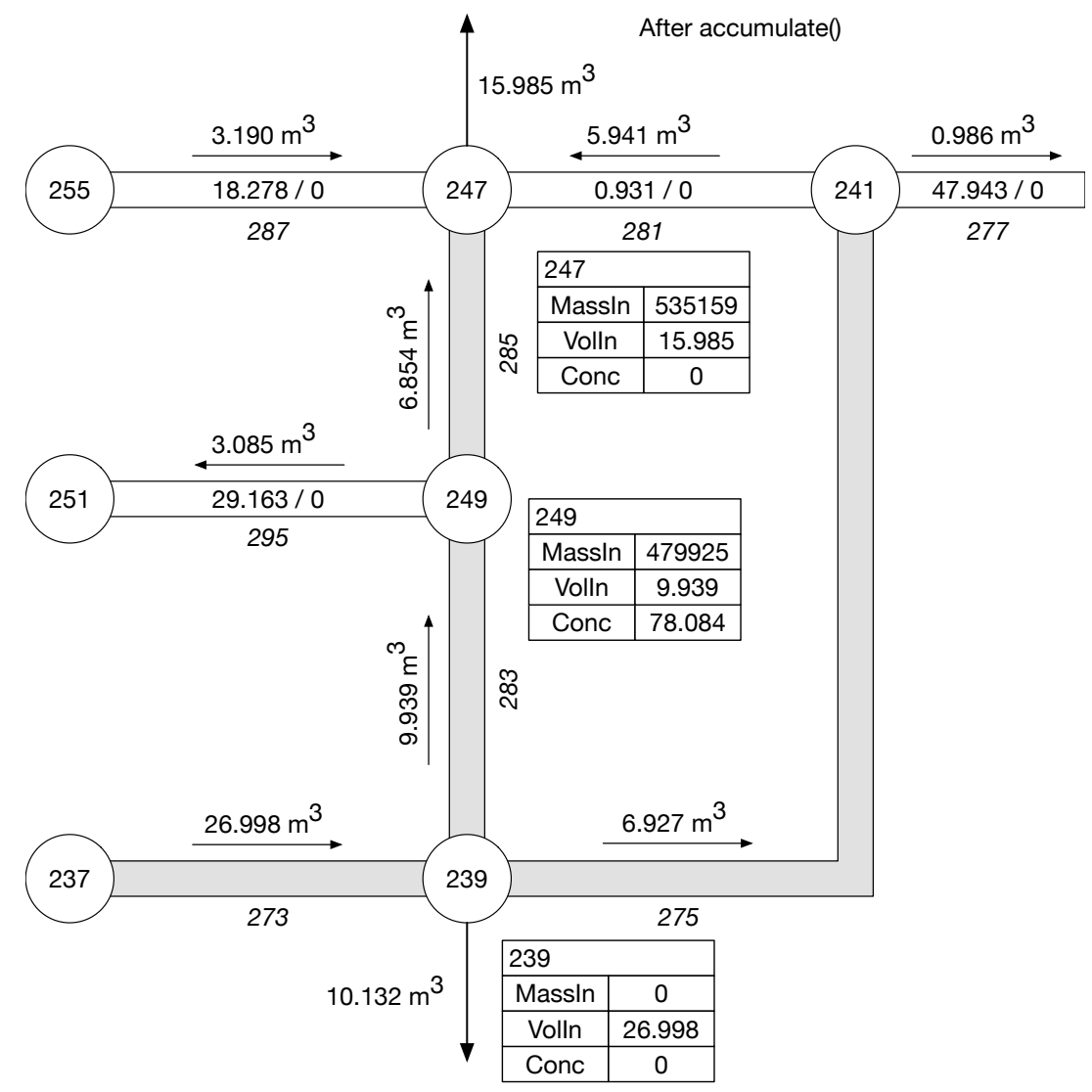

Figure B2. Example: conditions after accumulate().

demands from Node 247 (about $0.54 \mathrm{~kg}, 15.985 \mathrm{~m}^{3}$ of water with a constituent concentration of $33.479 \mathrm{mg} \mathrm{L}^{-1}$ ) and the mass in Links 285 and 295 (about $0.16 \mathrm{~kg}$ ), the MBR for the network at this point is about 1.4 ( 0.54 plus 0.16 divided by $0.5)$. The values for MassIn and VolIn shown in the tables in Fig. B4 are retained from the updatenodes step; they have no meaning at this point and are reset to zero at the beginning of the next time step.

\section{B3 Discussion}

As the example presented here illustrates, the accumulate step can result in the generation of constituent mass and the release step can result in the loss of mass. The condition necessary (but not sufficient) for generating or losing mass is a link with a volume less than the volume of water being moved in a water-quality time step. If the concentration of a water-quality constituent present in the network does not vary spatially, the mass generated in the accumulate step will equal the mass lost in the release step and there will be no net change in mass. However, if there is a spatial gradient in concentration so that the concentration of the excess volume generated during the accumulate step is different from the concentration associated with the volume of water lost in the release step, a net change in mass can occur. The net change can be either positive or negative: net mass can be generated or lost.

Let $V_{\mathrm{L}}$ be the volume of a link, $V_{\mathrm{M}}$ be the volume of water moved in the link during a water-quality time step, and $V_{\mathrm{M}}$ be greater than $V_{\mathrm{L}}$. Let $C_{\mathrm{A}}$ be the concentration of a constituent used for the extra volume for this link added in the accumulate step and $C_{\mathrm{R}}$ be the concentration of the constituent used for the lost volume for this link in the release step. Then the net change in constituent mass $(\Delta M)$ for the link for the time step is given by

$$
\begin{aligned}
\Delta M & =C_{\mathrm{A}}\left(V_{\mathrm{M}}-V_{\mathrm{L}}\right)-C_{\mathrm{R}}\left(V_{\mathrm{M}}-V_{\mathrm{L}}\right) \\
& =\left(C_{\mathrm{A}}-C_{\mathrm{R}}\right)\left(V_{\mathrm{M}}-V_{\mathrm{L}}\right) .
\end{aligned}
$$

Note that this relationship applies only when $V_{\mathrm{M}}>V_{\mathrm{L}}$. The volume of water being moved in a time step is $Q T$, where $Q$ is the flow rate on the link for the time step and $T$ is the length of the water-quality time step. Then,

$$
\Delta M=\left(C_{\mathrm{A}}-C_{\mathrm{R}}\right)\left(Q T-V_{\mathrm{L}}\right) .
$$




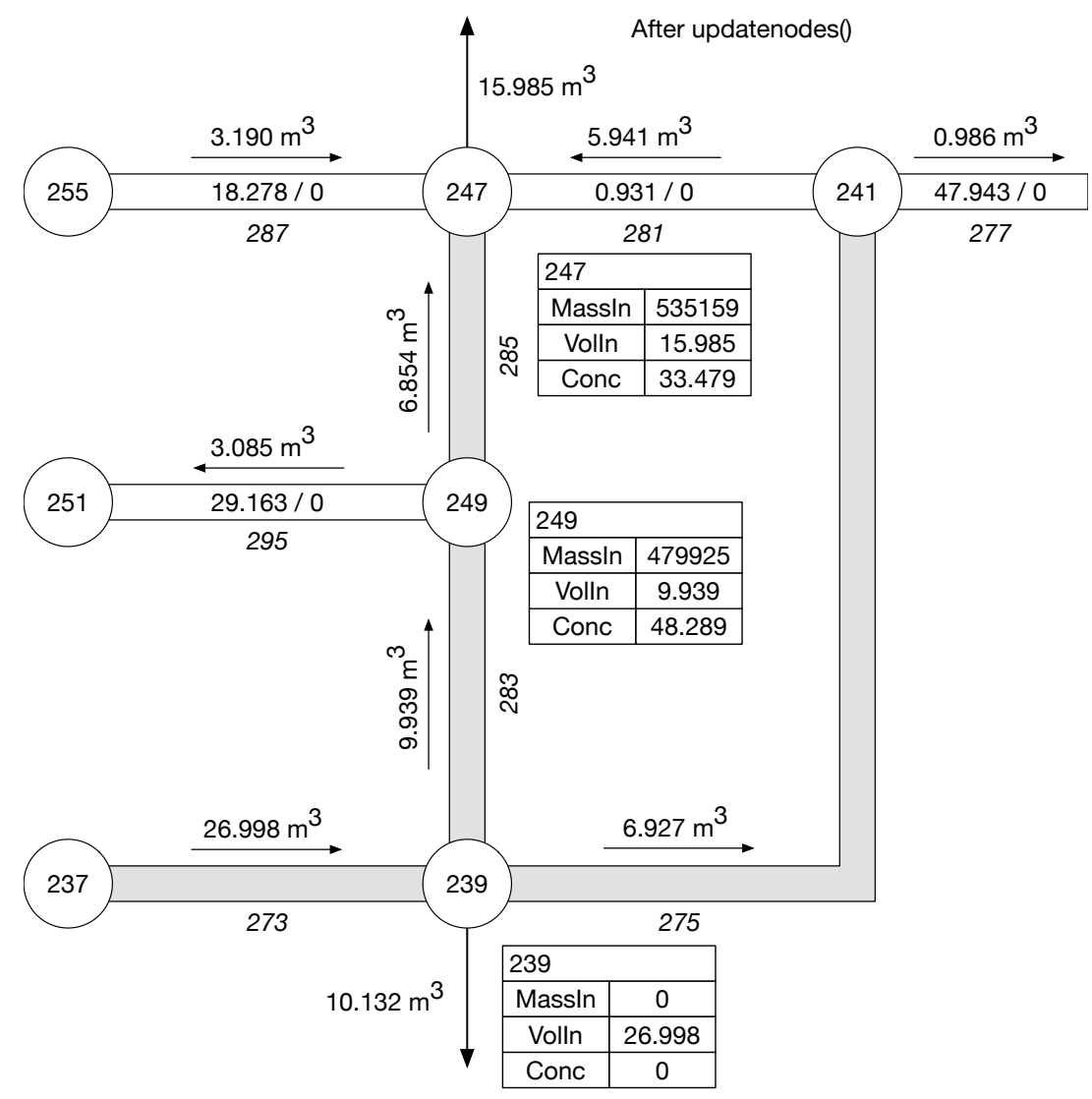

Figure B3. Example: conditions after updatenodes().

The volume of a link can be small; for a valve or pump it is zero. When the link volume is very small relative to $V_{M}$ the mass change for the link for the time step is proportional to the difference between the concentrations for the accumulate and release steps, the flow rate, and the water-quality time step. As the water-quality time step becomes small, the net mass change also become small.

For the example presented in this appendix, $C_{\mathrm{A}}=$ $78.084 \mathrm{mg} \mathrm{L}^{-1}$ and $C_{\mathrm{R}}=48.289 \mathrm{mg} \mathrm{L}^{-1}$. The difference between the volume moved in Link 285 and the link volume is $6.854 \mathrm{~m}^{3}-0.222 \mathrm{~m}^{3}=6.632 \mathrm{~m}^{3}$. Therefore, using Eq. (B1), the value for $\Delta M$ is (78.084$48.289)(6.632) / 1000=0.198 \mathrm{~kg}$. For a net mass gain of this amount the MBR is about 1.4, as noted above. The flow rate on Link 285 is small, $6.685 \mathrm{~m}^{3}$ per hour or about $0.002 \mathrm{~m}^{3} \mathrm{~s}^{-1}$. If the flow rate were an order of magnitude larger, the net mass gain would about $2 \mathrm{~kg}$ and the MBR would be about 5 . Substantial net mass changes are possible for a single link for a single water-quality time step. Note that when $C_{\mathrm{R}}>C_{\mathrm{A}}$, net mass losses will occur and the MBR will be less than 1 .

\section{Appendix C: Example using the event-driven approach}

This appendix presents a simple example to illustrate the application of the event-driven algorithm. The example also illustrates how the algorithm addresses cases with recirculating flows. The network used in the example is shown in Fig. C1. It has five nodes (A to E) with connecting links (labeled A-B, for example); Link D-E is a pump. Details of the network downstream (to the right) of Node D are not shown in the figure. There are demands at Nodes B, C, and E. The volumes shown above the links are the volumes of water moved during a water-quality time step; the arrows show the direction of flow. For example, $76 \mathrm{~m}^{3}$ of water are moved from Link A-B to Node B and $20 \mathrm{~m}^{3}$ of water are removed by demands at Node B during the time step. Each link can have one or more water parcels, which are volumes of water with uniform concentrations. The volumes of the parcels and the concentrations of a water-quality constituent present in the parcels are shown; for example, in Link E-B there are two parcels, one has a volume of $1700 \mathrm{~m}^{3}$ and a concentration of zero (labeled as 1700/0) and the second has a volume of $100 \mathrm{~m}^{3}$ and a concentration of $10 \mathrm{mg} \mathrm{L}^{-1}$ (labeled as 


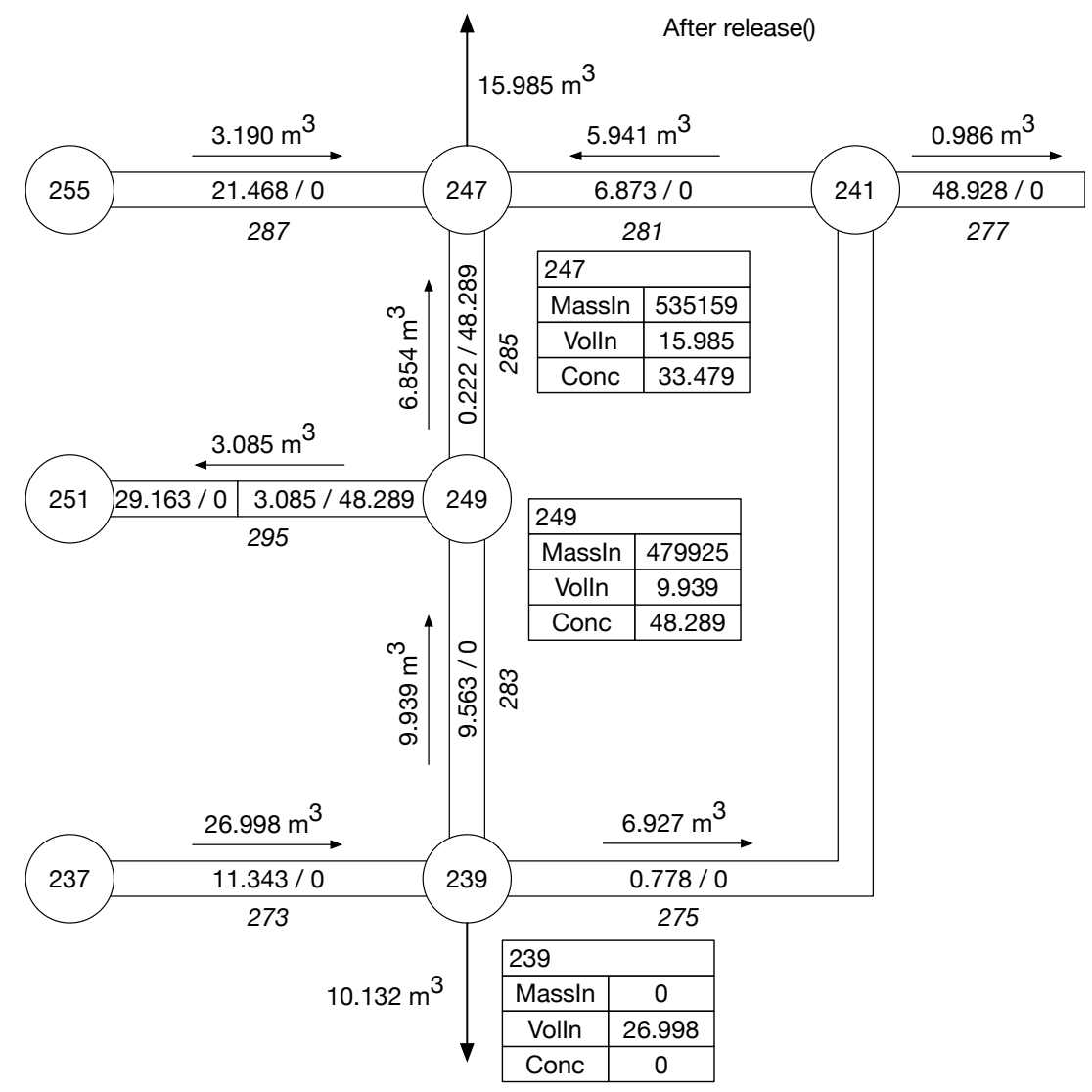

Figure B4. Example: conditions after release().

100/10). The volume of a link equals the sum of the volumes of water parcels in the link. For example, Link E-B has a volume of $1800 \mathrm{~m}^{3}$, equal to the sum of $100 \mathrm{~m}^{3}$ plus $1700 \mathrm{~m}^{3}$. Note that the volume of Link D-E, a pump, is zero. The quality tolerance is $0.01 \mathrm{mg} \mathrm{L}^{-1}$; adjacent water parcels whose concentrations differ by less than this amount are combined.

As shown in Fig. C1, there is an inflow to Node B from Link A-B with a concentration of zero. There is also an inflow from Link E-B; however, the concentration for that inflow has not yet been determined and the inflow cannot be processed. Consequently, the state of the inflow from Link E-B is labeled as $4 /$ ? in the small table in the figure, indicating that the concentration has not yet been determined. It may seem obvious that the concentration of the inflow from Link E-B should be $10 \mathrm{mg} \mathrm{L}^{-1}$, given that this is the concentration of the leading parcel in the link. However, in general, the concentration of the inflow from Link E-B will be affected by the volume of the inflow. More than one water parcel might need to be combined to provide the needed inflow volume, and the operations required to determine if any combining of parcels is necessary and to determine the resulting concentration have not yet been carried out. Therefore, to accommodate this situation, an incomplete parcel representing the merging of inflows from Links A-B and E-B is created that has an unspecified concentration but a known volume. This incomplete parcel maintains references to the parcels that merged to form it. The parcel (called Seg1) is then moved through Node B with the result shown in Fig. C2, which illustrates how water parcels from Links A-B and E$B$ are combined at Node B to form Parcel Seg1, which is then split into Parcels Seg2 and Seg3 to satisfy the nodal demand and the outflow from the node. Node B's demand list has a reference to Parcel Seg2, which shows that of the parcel's total volume of $20 \mathrm{~m}^{3}$, the parcel from Link A-B contributed $19 \mathrm{~m}^{3}$ and the parcel from Link E-B contributed $1 \mathrm{~m}^{3}$; the ratio of the contributions to Parcel Seg2 (and Seg3) is 19:1, the same as the ratio of inflows from Links A-B and E-B. Parcel Seg4 is created and added as the trailing segment in Link B-C. After Parcel Seg4 is added to Link B-C, $60 \mathrm{~m}^{3}$ of water are removed from the leading water parcel in the link; the leading parcel in the link then has a volume of $740 \mathrm{~m}^{3}$ (and a concentration of zero, shown as 740/0 in Fig. C2), maintaining the total volume of the parcels in the link equal to the link's volume, namely $800 \mathrm{~m}^{3}$. 


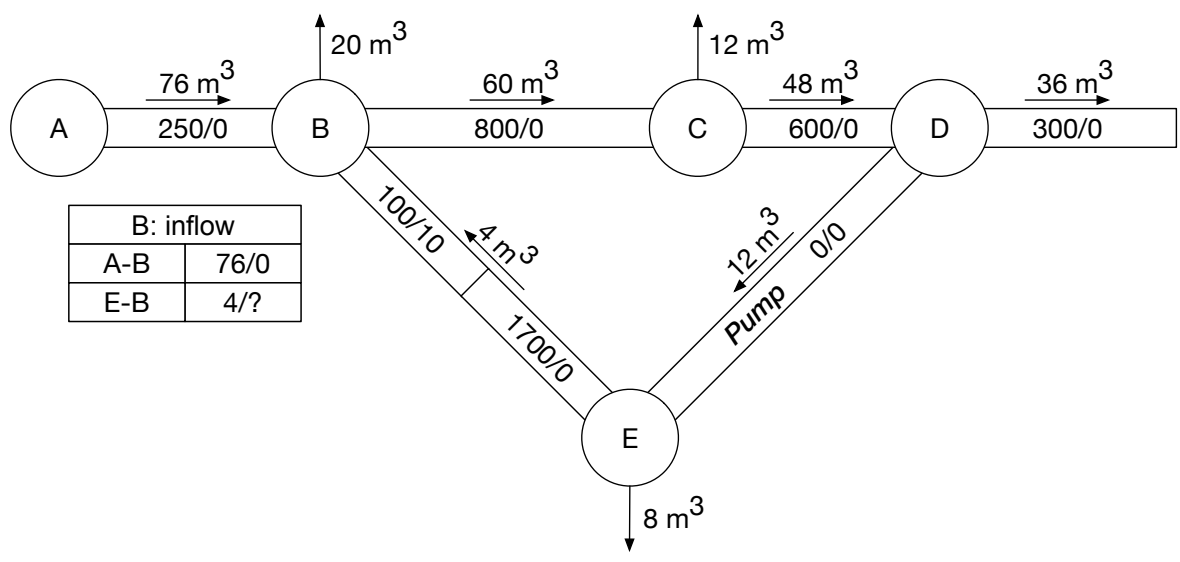

Figure C1. Example: network at the beginning of the time step. (The $36 \mathrm{~m}^{3}$ of water leaving Node D goes to a downstream portion of the network not shown in the figure.)

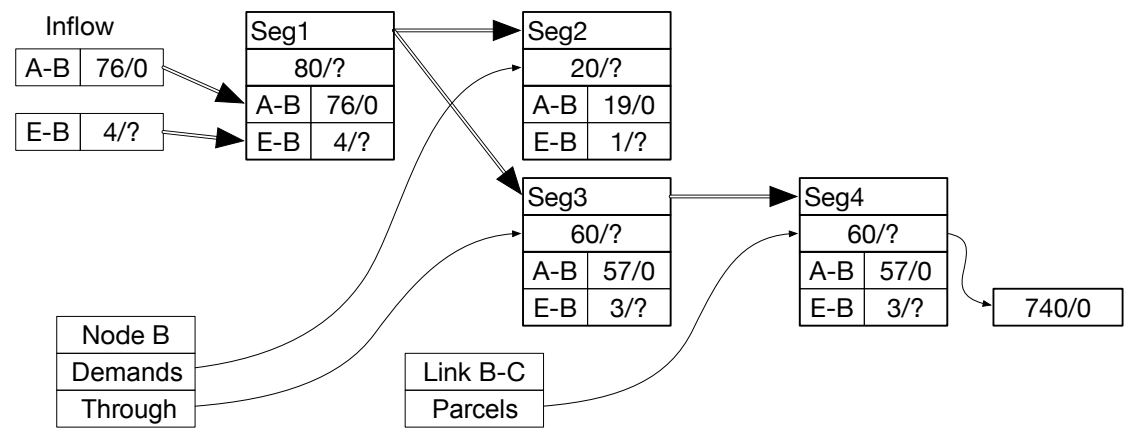

Figure C2. Example: creation of incomplete water parcels.

At this point Parcel Seg1 is no longer referenced directly by any link or node. However, it has internal references so that when the concentration of the water parcel arriving at Node B from Link E-B has been determined, the parcel can be completed. When the parcel is completed, the result will cascade to its children, namely Seg2 and Seg3.

Adding Parcel Seg4 with a volume of $60 \mathrm{~m}^{3}$ to Link B-C results in the same volume of water being moved to Node $\mathrm{C}$ from the leading parcel on Link B-C. An amount of $12 \mathrm{~m}^{3}$ of water are removed from that parcel and placed on Node C's demand list; the remaining $48 \mathrm{~m}^{3}$ of water from the parcel are added to Link C-D. At Node D, $48 \mathrm{~m}^{3}$ of water are removed from the link, with the parcel that is removed being split: $36 \mathrm{~m}^{3}$ leave Node D to be moved through the rest of the network (not shown) and $12 \mathrm{~m}^{3}$ are placed on the trailing end of Link D-E. At Node E, $8 \mathrm{~m}^{3}$ of water are removed and placed on Node E's demand list. A parcel with a volume of $4 \mathrm{~m}^{3}$ is added at the trailing end of Link E-B, which causes $4 \mathrm{~m}^{3}$ of the leading parcel in Link E-B (with a concentration of $10 \mathrm{mg} \mathrm{L}^{-1}$ ) to be sent to Node B. Because the concentration of the $4 \mathrm{~m}^{3}$ parcel added to Link E-B is the same (zero) as the concentration of the trailing $1700 \mathrm{~m}^{3}$ parcel, the two are combined to yield a parcel with a volume of $1704 \mathrm{~m}^{3}$ and a concentration of zero. The situation at this point is shown in Fig. C3.

When the leading parcel from Link E-B arrives at Node B, incomplete Parcel Seg1 can be completed because its concentration can now be determined. The concentrations of all the other incomplete parcels shown in Fig. C2 also can be determined and updated values for these concentrations are shown in Fig. C4. The concentration of Parcel Seg1 $\left(0.5 \mathrm{mg} \mathrm{L}^{-1}\right)$ is the concentration of the blended parcels coming from Link $\mathrm{A}-\mathrm{B}\left(76 \mathrm{~m}^{3}\right.$ with a concentration of 0$)$ and Link E-B $\left(4 \mathrm{~m}^{3}\right.$ with a concentration of $\left.10 \mathrm{mg} \mathrm{L}^{-1}\right)$. Parcels Seg2, Seg3, and Seg4 have the same concentration. The status of the network at the end of the time step is shown in Fig. C5.

In general, in applications using the event-driven algorithm there are multiple interim steps within each waterquality time step. Each of these steps corresponds to an event in the simulation. The preceding discussion mentions the various interim steps included in the time step considered, but 


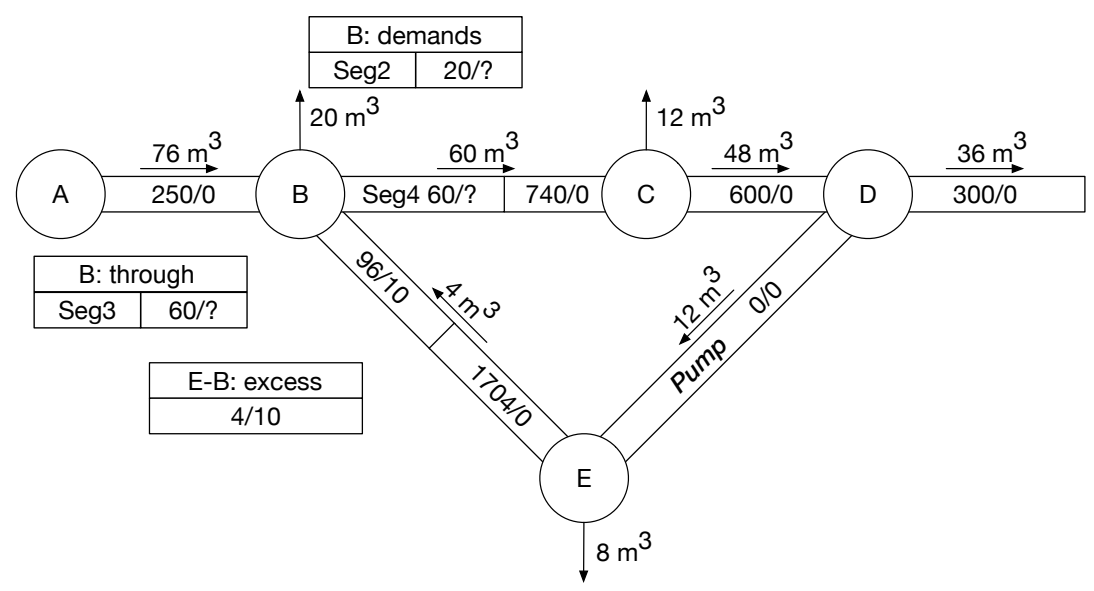

Figure C3. Example: interim status of the network.

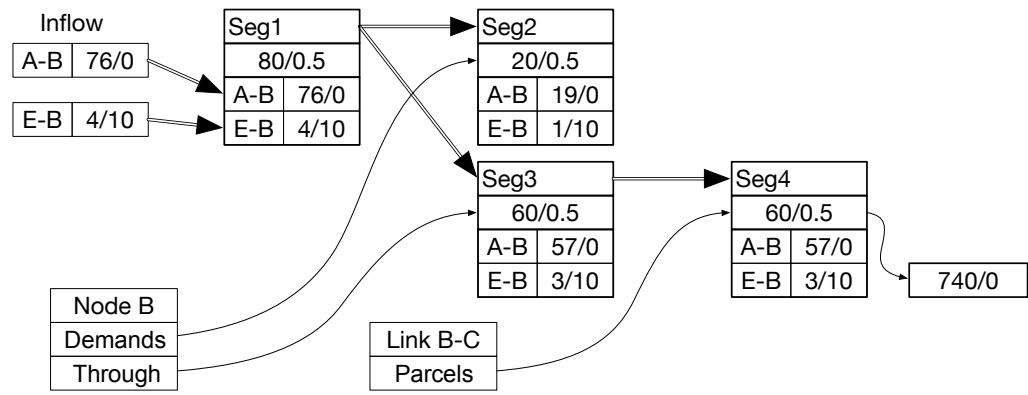

Figure C4. Example: completion of incomplete water parcels.

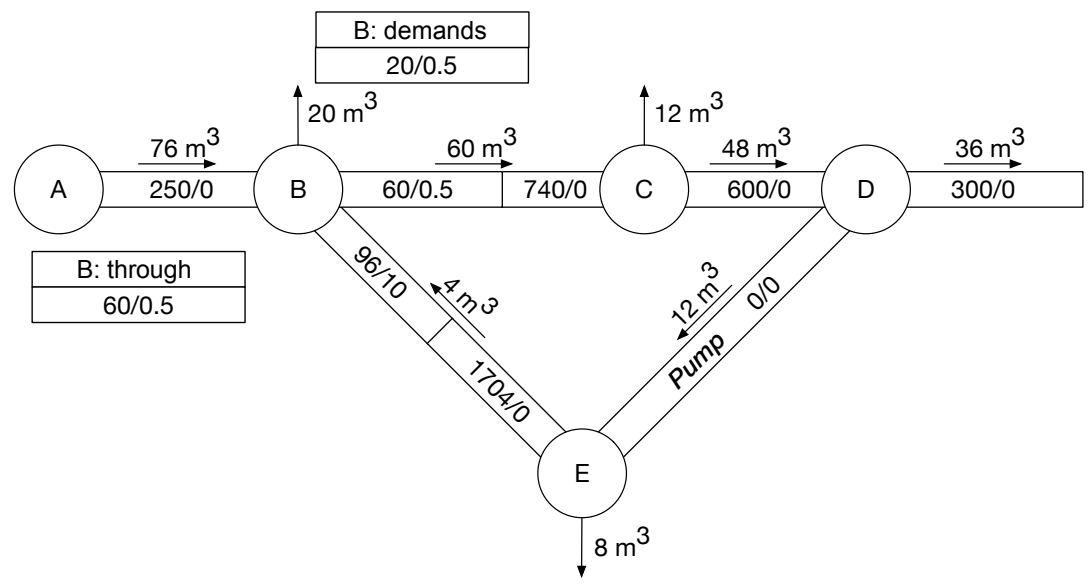

Figure C5. Example: network at the end of the time step. 
Table C1. Water-quality routing for the example using the event-driven approach. Entries in the table describe water parcels, giving their volume and concentration (volume/concentration). Results are shown for one water-quality time step. The last row in the table gives the volume of water moved in a time step.

\begin{tabular}{|c|c|c|c|c|c|c|c|c|}
\hline \multirow[b]{2}{*}{ Time or volume } & \multicolumn{8}{|c|}{ Location $^{\mathrm{a}, \mathrm{b}}$} \\
\hline & A-B & $D_{\mathrm{B}}$ & $\mathrm{B}-\mathrm{C}$ & $D_{\mathrm{C}}$ & C-D & D-E & $D_{\mathrm{E}}$ & E-B \\
\hline Time $=0$ & $250 / 0$ & $20 / ?$ & $800 / 0$ & $12 / ?$ & $600 / 0$ & $0 / 0$ & $8 / ?$ & $1700 / 0,100 / 10$ \\
\hline Step 1 & $76 / 0,250 / 0$ & $20 / ?$ & $800 / 0$ & $12 / ?$ & $600 / 0$ & $0 / 0$ & $8 / ?$ & $1700 / 0,100 / 10$ \\
\hline Step $2^{\mathrm{c}}$ & $250 / 0$ & $19 / 0,1 / ?$ & $57 / 0,3 / ?, 800 / 0$ & $12 / ?$ & $600 / 0$ & $0 / 0$ & $8 / ?$ & $1700 / 0,100 / 10$ \\
\hline Step 3 & $250 / 0$ & $19 / 0,1 / ?$ & $57 / 0,3 / ?, 740 / 0$ & $12 / 0$ & $48 / 0,600 / 0$ & $0 / 0$ & $8 / ?$ & $1700 / 0,100 / 10$ \\
\hline Step 4 & $250 / 0$ & $19 / 0,1 / ?$ & $57 / 0,3 / ?, 740 / 0$ & $12 / 0$ & $600 / 0$ & $12 / 0,0 / 0$ & $8 / ?$ & $1700 / 0,100 / 10$ \\
\hline Step 5 & $250 / 0$ & $19 / 0,1 / ?$ & $57 / 0,3 / ?, 740 / 0$ & $12 / 0$ & $600 / 0$ & $0 / 0$ & $8 / 0$ & $4 / 0,1700 / 0,100 / 10$ \\
\hline Step 6 & $250 / 0$ & $19 / 0,1 / 10$ & $57 / 0,3 / 10,740 / 0$ & $12 / 0$ & $600 / 0$ & $0 / 0$ & $8 / 0$ & $1704 / 0,96 / 10$ \\
\hline Time $=$ a time step & $250 / 0$ & $20 / 0.5$ & $60 / 0.5,740 / 0$ & $12 / 0$ & $600 / 0$ & $0 / 0$ & $8 / 0$ & $1704 / 0,96 / 10$ \\
\hline Vol. moved $\left(\mathrm{m}^{3}\right)$ & 76 & 20 & 60 & 12 & 48 & 12 & 8 & 4 \\
\hline
\end{tabular}

a A-B, etc are links. $D_{\mathrm{B}}$, etc are nodal demands. ${ }^{\mathrm{b}}$ A question mark (?) means that the concentration has not yet been determined. ${ }^{\mathrm{c}}$ The two parcels in $D_{\mathrm{B}}$ correspond to Seg2 in Fig. C2 and the two trailing parcels in B-C correspond to Seg3 in the same figure. Seg1 in the figure corresponds to an intermediate step before the creation of Seg2 and Seg3. It is not associated with any link or demand and therefore is not included in this table.

emphasizes the method used to address situations involving a recirculating flow. It does not focus explicitly on the various interim steps themselves. The interim steps in the example are provided explicitly in Table $\mathrm{C} 1$, which shows event by event the changes that take place during the time step considered in the example.

The columns in Table $\mathrm{C} 1$ correspond to either a link in the network used in the example or to a demand at one of its nodes. The rows correspond to the steps used in the algorithm to route water quality through the network. In this example there are six interim steps between the beginning and end of the water-quality time step. The entries in the table correspond to water parcels. The same notation used above to describe a water parcel is used in the table. For example, $250 / 0$ in the first row of the first column indicates that there is a water parcel with a volume of $250 \mathrm{~m}^{3}$ and a concentration of zero in Link A-B at the beginning of the time step. More than one entry indicates that there is more than one water parcel associated with the link or demand at that specific step in the process. The leading parcel in each entry is the rightmost one.
In Step 1, a water parcel (76/0) is added to Link A-B due to the inflow of $76 \mathrm{~m}^{3}$ of water to the link from Node A. The concentration of the new parcel is the same (zero) as the concentration of the parcel already in the link (250/0) and the two parcels are combined. Because the combined volume of the parcels now exceeds the volume of the link, the excess volume is moved (Step 2) to Node B, where it is combined with a water parcel from Link E-B (4/?) and then split to accommodate the demand for Node $\mathrm{B}\left(D_{\mathrm{B}}\right.$ in the table) and outflow for the node. As discussed above, the concentration for the parcel arriving from Link E-B has not yet been determined, so incomplete parcels must now be used. Excess water is moved step by step through the network until it is removed by downstream nodes (at Node $D$ ) or by demands. By Step 5 the concentration of the inflow from Link E-B can be determined and the incomplete parcels can be completed (Step 6). By the end of the time step the water added at the beginning of the time step has moved through the network and volumes and concentrations of all water parcels and demands have been determined. The conditions at the beginning of the time step correspond to those in Fig. $\mathrm{C} 1$ and those at the end of the time step correspond to those in Fig. C5. The conditions in Step 2 correspond to those in Fig. C2 and those in Step 6 correspond to those in Fig. C4. 
Competing interests. The authors declare that they have no conflict of interest.

Disclaimer. This paper has been subjected to EPA's review and has been approved for publication. The views expressed in this paper are those of the authors and approval does not signify that the contents necessarily reflect the views of the Agency. Mention of trade names, products, or services does not convey official EPA approval, endorsement, or recommendation. Because of the confidentiality of the information, the identity of the real WDSs used in this paper and any information that could be used to identify the systems cannot be disclosed.

The submitted manuscript has been created by UChicago Argonne, LLC, operator of Argonne National Laboratory ("Argonne"). Argonne, a US Department of Energy Office of Science laboratory, is operated under contract no. DE-AC02-06CH11357. The US government retains for itself, and others acting on its behalf, a paid-up nonexclusive, irrevocable worldwide license in said article to reproduce, prepare derivative works, distribute copies to the public, and perform publicly and display publicly, by or on behalf of the government. The Department of Energy will provide public access to these results of federally sponsored research in accordance with the DOE Public Access Plan (http://energy.gov/ downloads/doe-public-access-plan).

Acknowledgements. The US Environmental Protection Agency's (EPA) Office of Research and Development funded, managed, and participated in the research described here under an interagency agreement. Work at Argonne National Laboratory was sponsored by the EPA under an interagency agreement through US Department of Energy Contract DE-AC02-06CH11357. All post-simulation data analysis and preparation of graphics for this paper were done using $\mathrm{R}$ (R Core Team, 2017). Network plots were produced in $\mathrm{R}$ using epanetReader (Eck, 2016). Four internal reviewers provided helpful comments on a draft version of this paper. We also appreciate the helpful comments provided by Patrick Moore and Sam Hatchett during the interactive discussion and the helpful comments from two anonymous referees.

Edited by: Ran Shang

Reviewed by: two anonymous referees

\section{References}

Boulos, P. F., Altman, T., Jarrige, P.-A., and Collevati, F.: An event-driven method for modelling contaminant propagation in water networks, Appl. Math. Model., 18, 84-92, https://doi.org/10.1016/0307-904X(94)90163-5, 1994.

Boulos, P. F., Altman, T., Jarrige, P.-A., and Collevati, F.: Discrete simulation approach for network-waterquality models, J. Water Res. Pl.-ASCE, 121, 49-60, https://doi.org/10.1061/(ASCE)0733-9496(1995)121:1(49), 1995.

Brumbelow, K., Torres, J., Guikema, S., Bristow, E., and Kanta, L.: Virtual cities for water distribution and infrastructure system research, Proc. World Environ. and Water Res. Congress 2007,
ASCE, Reston, VA, https://doi.org/10.1061/40927(243)469, 2007.

Davis, M. J. and Janke, R.: Influence of network model detail on estimated health effects of drinking water contamination events, J. Water Res. Pl.-ASCE, 141, https://doi.org/10.1061/(ASCE)WR.1943-5452.0000436, 2014.

Davis, M. J., Janke, R., and Magnuson, M. L.: A framework for estimating the adverse health effects of contamination events in water distribution systems and its application, Risk Anal., 34, 498-513, https://doi.org/10.1111/risa.12107, 2014.

Davis, M. J., Janke, R., and Taxon, T. N.: Assessing inhalation exposures associated with contamination events in water distribution systems, PLoS ONE, 11, e0168051, https://doi.org/10.1371/journal.pone.0168051, 2016.

Diao, K., Sweetapple, C., Farmani, R., Fu, G., Ward, S., and Butler, D.: Global resilience analysis of water distribution systems, Water Res., 106, 383-393, https://doi.org/10.1016/j.watres.2016.10.011, 2016.

Eck, B. J.: An $\mathrm{R}$ package for reading EPANET files, Environ. Modell. Softw., 84, 149-154, https://doi.org/10.1016/j.envsoft.2016.06.027, 2016.

GCC: GCC, the GNU compiler collection, available at: https://gcc. gnu.org, last access: 19 April 2017.

Helbling, D. E. and VanBriesen, J. M.: Propagation of chlorine signals induced by microbial contaminants in a drinking water distribution system, Proc. World Environ. and Water Res. Congress 2009: Great Rivers, ASCE, Kansas City, MO, 515524, https://doi.org/10.1061/41036(342)50, 2009.

OpenWaterAnalytics: EPANET, available at: https://github.com/ OpenWaterAnalytics/EPANET, last access: 16 August 2017a.

OpenWaterAnalytics: epanet-dev, available at: https://github.com/ OpenWaterAnalytics/epanet-dev, last access: 16 August 2017b.

Ostfeld, A., Uber, J. G., Salomons, E., Berry, J. W., Hart, W. E., Phillips, C. A., Watson, J-P., Dorini, G., Jonkergouw, P., Kapelan, Z., di Pierro, F., Khu, S-T., Savic, D., Eliades, D., Polycarpou, M., Ghimire, S. R., Barkdoll, B. D., Gueli, R., Huang, J. J., McBean, E. A., James, W., Krause, A., Leskovec, J., Isovitsch, S., Xu, J., Guestrin, C., VanBriesen, J., Small, M., Fischbeck, P., Preis, A., Propato, M., Piller, O., Trachtman, G. B., Wu, Z. Y., and Walski, T.: The battle of the water sensor networks (BWSN): A design challenge for engineers and algorithms, J. Water Res. Pl.-ASCE, 134, 556-568, https://doi.org/10.1061/(ASCE)07339496(2008)134:6(556), 2008.

R Core Team: R: A language and environment for statistical computing, R Foundation for Statistical Computing, Vienna, Austria, available at: https://www.R-project.org, last access: $3 \mathrm{Au}$ gust 2017.

Rossman, L. A.: EPANET 2 users manual, US Environmental Protection Agency (EPA), Office of Research and Development, National Risk Management Research Laboratory, Cincinnati, Ohio, EPA/600/R-00/057, 2000.

Rossman, L. A. and Boulos, P. F.: Numerical methods for modeling water quality in distribution systems: A comparison, J. Water Res. Pl.-ASCE, 122, 137-146, https://doi.org/10.1061/(ASCE)0733-9496(1996)122:2(137), 1996.

Taxon, T.: Flow method for water quality transport, available at: https://github.com/ttaxon/EPANET/tree/flow-transport-model, last access: 26 March 2018. 
US EPA: EPANET: Software that models the hydraulic and water quality behavior of water distribution piping systems, available at: https://www.epa.gov/water-research/epanet, last access: 16 August 2017a.

US EPA: Water-distribution-network-model, available at: https://github.com/USEPA/Water-Distribution-Network-Model, last access: 16 August 2017b.

US EPA: Models, tools and applications for homeland security research, available at: https://www.epa.gov/homeland-security-research/ models-tools-and-applications-homeland-security-research, last access: 16 August 2017c.

US EPA: EPANET INP files used in paper, https://doi.org/10.23719/1375314, 2018a.
US EPA: TEVA-SPOT-GUI - Containing preliminary flow model, https://doi.org/10.23719/1375315, 2018b.

Wang, H. and Harrison, K. W.: Improving efficiency of the Bayesian approach to water distribution contaminant source characterization with support vector regression, J. Water Res. Pl.-ASCE, 140, 3-11, https://doi.org/10.1061/(ASCE)WR.19435452.0000323, 2014.

Wu, Z. Y.: Heterogeneous computing paradigm for parallel water distribution system analysis, CUNY Academic Works, available at: http://academicworks.cuny.edu/cc_conf_hic/91 (last access: 15 February 2018), 2014. 